\title{
Quasi-convergence of model geometries under the Ricci flow
}

\begin{abstract}
Dan Knopf and Kevin McLeod
We study the quasi-convergence equivalence classes of all locally homogeneous metrics for which the Ricci flow exists to infinity. We consider the most general possible families of such metrics by using model geometries $(\mathcal{X}, \mathcal{G})$ with $\mathcal{G}$ minimal.
\end{abstract}

\section{Introduction.}

There are many Riemannian 3-manifolds $\left(\mathcal{M}^{3}, g\right)$ for which the Ricci flow

$$
\frac{\partial}{\partial t} g=-2 \mathrm{Rc}
$$

exists for all positive time, yet fails to converge. Many of these examples collapse, hence are not explicitly studied in the classification [3] of nonsingular solutions. (We say a solution to the Ricci flow collapses if the maximum injectivity radius of the corresponding solution to the normalized Ricci flow

$$
\frac{\partial}{\partial t} g=-2 \mathrm{Rc}+2 \frac{\int R}{\int n} g
$$

goes to 0 as $t \rightarrow \infty$.) Hamilton has conjectured that the large-time behavior of any collapsing solution will in some sense approach the evolution of a locally homogeneous geometry. (Compare [5].) This idea can be developed by using the concept of quasi-convergence, an equivalence relation which allows us to classify the asymptotic behavior of the Ricci flow. Recall [6] that if $g, h$ are evolving Riemannian metrics on a manifold $\mathcal{M}^{n}$, we say $g$ quasi-converges to $h$ and write $g \in[h]$ if for any $\varepsilon>0$ there is a time $t_{\varepsilon}$ such that

$$
\sup _{\mathcal{M}^{n} \times\left[t_{\varepsilon}, \infty\right)}|g-h|_{h}<\varepsilon .
$$

From the standpoint of geometry, this might seem like an unduly restrictive definition, because it does not allow modification by diffeomorphisms. 
But from the standpoint of analysis, it describes the large-time behavior of the Ricci flow more precisely than if, say, we considered $\left|\phi_{i}^{*} g_{i}-h_{i}\right|_{h_{i}}$ for sequences of metrics $g_{i}, h_{i}$ and arbitrary diffeomorphisms $\phi_{i}$.

Hamilton-Isenberg [4] introduced the term 'quasi-convergence' in their study of a particular class of metrics on solv-twisted torus bundles. The definition was formulated in [6] as part of an effort to refine our understanding of the asymptotic behavior of metrics in that class. The intent of the current paper is to broaden that investigation and to complement the work of Isenberg-Jackson [5] by determining the quasi-convergence equivalence classes of all locally homogeneous metrics for which the Ricci flow exists for all time.

Since a locally homogeneous metric on a simply-connected manifold is homogeneous [9] and the Ricci flow commutes with covering projections, it suffices to study model geometries. A model geometry [10], [8] is a pair $(\mathcal{X}, \mathcal{G})$, where $\mathcal{X}$ is a simply-connected smooth manifold and $\mathcal{G}$ is a group of diffeomorphisms which acts transitively on $\mathcal{X}$ with compact point stabilizers $\mathcal{G}_{x}$. Given any $\mathcal{G}_{x}$-invariant scalar product on $T_{x} \mathcal{X}$ for some (hence any) $x \in \mathcal{X}$, we obtain in a natural way a complete homogeneous $\mathcal{G}$-invariant metric on $\mathcal{X}$. For the purpose of describing canonical Riemannian metrics on the eight geometric structures of the Thurston conjecture, it is desirable that $\mathcal{G}$ (hence $\mathcal{G}_{x}$ ) be as large as possible. But for our purpose of classifying the asymptotic behavior of the Ricci flow, we have chosen smaller transitive groups in order to study larger families of metrics. For each model that we study, we are able to consider a full 6-dimensional family of metrics by finding a realization $(\mathcal{X}, \mathcal{G})$ with trivial stabilizers $\mathcal{G}_{x}$. We shall say each such family has the geometry of the canonical metric it contains. Five of Thurston's eight models can be realized by the pair $(\mathcal{G}, \mathcal{G})$, where $\mathcal{G}$ is a simply-connected unimodular Lie group. There are exactly six such groups, and they have been studied by Milnor. [7] $\left(\mathbb{R}^{3}\right.$ and Isom $\left(\mathbb{E}^{2}\right)$ both have the geometry of $\mathbb{E}^{3}$.) The three remaining models $\left(\mathbb{H}^{3}, \mathbb{H}^{2} \times \mathbb{E}^{1}\right.$, and $\left.\mathbb{S}^{2} \times \mathbb{E}^{1}\right)$ must be treated separately.

Our results are summarized in Table 1 . (Here, $\mathcal{N}^{3}$ denotes the Heisenberg group, $\mathcal{H}^{n}$ denotes the upper half-space in $\mathbb{R}^{n}$, and $\mathcal{I}^{n}$ is a transitive subgroup of Conf $\left(\mathcal{H}^{n}\right)$ defined in $\S 3$.) Notice the inverse relationship between the size of the isotropy group under which the canonical metric in each family is invariant and the size of the quasi-convergence equivalence class of an arbitrary metric in that family. 


\begin{tabular}{|c|c|c|c|}
\hline Geometry & Isotropy & Realization $(\mathcal{X}, \mathcal{G})$ & Class $[g]$ \\
\hline $\mathbb{S}^{3}$ & $\mathrm{O}(3)$ & $\mathrm{SU}(2), \mathrm{SU}(2)$ & singularity at $T<\infty$ \\
\hline $\mathbb{E}^{3}$ & $\mathrm{O}(3)$ & $\mathbb{R}^{3}, \mathbb{R}^{3}$ & 0-dimensional \\
\hline $\mathbb{H}^{3}$ & $\mathrm{O}(3)$ & $\mathcal{H}^{3}, \mathcal{I}^{3}$ & 1-dimensional \\
\hline $\mathbb{S}^{2} \times \mathbb{E}^{1}$ & $\mathrm{O}(2)$ & $\mathbb{R} \times \mathbb{S}^{2}, \operatorname{Isom}(\mathbb{R}) \times \mathrm{SO}(3)$ & singularity at $T<\infty$ \\
\hline $\mathbb{E}^{3}$ & $\mathrm{O}(3)$ & $\widetilde{\operatorname{Isom}\left(\mathbb{E}^{2}\right)}$, Isom $\left(\mathbb{E}^{2}\right)$ & 2-dimensional \\
\hline $\mathbb{H}^{2} \times \mathbb{E}^{1}$ & $\mathrm{O}(2)$ & $\mathbb{R} \times \mathcal{H}^{2}, \operatorname{Isom}(\mathbb{R}) \times \mathcal{I}^{2}$ & 3-dimensional \\
\hline$\widehat{\mathrm{SL}(2, \mathbb{R})}$ & $\mathrm{O}(2)$ & $\dot{\mathrm{SL}(2, \mathbb{R})}, \widehat{\mathrm{SL}(2, \mathbb{R})}$ & $\geq 2$-dimensional \\
\hline nil & $\mathrm{O}(2)$ & $\mathcal{N}^{3}, \mathcal{N}^{3}$ & 3-dimensional \\
\hline solv & trivial & $\operatorname{Isom}\left(\mathbb{E}_{1}^{1}\right), \operatorname{Isom}\left(\mathbb{E}_{1}^{1}\right)$ & 4-dimensional \\
\hline
\end{tabular}

Table 1: Quasi-convergence in the model geometries.

A comment is in order concerning our (lack of) results for $\widetilde{\mathrm{SL}(2, \mathbb{R})}$. We have heuristic arguments which suggest that $[g]$ is 3-dimensional in this case, but have encountered two obstacles to the proof of this fact. Our analysis of the 'off-diagonal' sub-family of these metrics, while straightforward in principle, is computationally formidable, perhaps even intractable. A more serious obstacle, however, has been our inability to find any conserved quantity for arbitrary $\widehat{\mathrm{SL}(2, \mathbb{R})}$ metrics - in sharp contrast with the other geometries. Because of the close relationship between quasi-convergence and conservation laws, this forced us to rely on less effective qualitative analyses of their behavior.

\section{The geometries modeled by a Lie group.}

Let $\mathcal{G}^{n}$ be a Lie group, and let $\mathfrak{g}$ be the Lie algebra of all left-invariant vector fields on $\mathcal{G}$. Since a left-invariant metric on $\mathcal{G}$ is equivalent to a scalar product on $\mathfrak{g}$, the set of all such metrics can be identified with the set $\mathcal{S}_{n}^{+}$ of symmetric positive-definite $n \times n$ matrices. $\mathcal{S}_{n}^{+}$is an open convex subset of $\mathbb{R}^{n(n+1) / 2}$. For each left-invariant metric $g$ on $\mathcal{G}$, the Ricci flow may thus be regarded as a path $t \mapsto g(t) \in \mathcal{S}_{n}^{+}$.

Now consider a 3-dimensional unimodular simply-connected Lie group $\mathcal{G}$, and let $g, h \in \mathcal{S}_{3}^{+}$be arbitrary left-invariant metrics. It is impractical in general to study the system of six ODE which results when we wish to 
compare the evolution of $g$ and $h$ under the Ricci flow. Hence we analyze $[g]$ in two steps:

2.0.1. The diagonal case. For any left-invariant metric $g$ on $\mathcal{G}$, there is by [7] a left-invariant orthogonal frame field $\mathcal{F}=\left\{F_{i}\right\}$ such that the structure constants $c_{i j}^{k}$ defined by

$$
\left[F_{i}, F_{j}\right]=c_{i j}^{k} F_{k}
$$

all vanish except possibly when $i \neq j \neq k$. Indeed, if we define $\lambda^{i}$ by

$$
2 \lambda^{1} \doteqdot c_{23}^{1}, \quad 2 \lambda^{2} \doteqdot c_{31}^{2}, \quad 2 \lambda^{3} \doteqdot c_{12}^{3},
$$

we can arrange that $\lambda^{1} \leq \lambda^{2} \leq \lambda^{3} \in\{-1,0,1\}$. We call a frame field with these properties a Milnor frame for $g$. The signature of $\left(\lambda^{i}\right)$ is characteristic of the Lie algebra $\mathfrak{g}$ (hence of the unique simply-connected Lie group $\mathcal{G}$ associated to that algebra) according to Table 2.

\begin{tabular}{|cc|l|}
\hline$(-,-,-)$ & $(+,+,+)$ & $\mathcal{G}$ is $\mathrm{SU}(2)$. \\
$(-,-, 0)$ & $(0,+,+)$ & $\mathcal{G}$ is $\widetilde{\operatorname{Isom}\left(\mathbb{E}^{2}\right) .}$ \\
$(-,-,+)$ & $(-,+,+)$ & $\mathcal{G}$ is $\mathrm{SL}(2, \mathbb{R})$. \\
$(-, 0,0)$ & $(0,0,+)$ & $\mathcal{G}$ is the Heisenberg group. \\
$(-, 0,+)$ & $\mathcal{G}$ is Isom $\left(\mathbb{E}_{1}^{1}\right)$. \\
$(0,0,0)$ & $\mathcal{G}$ is $\mathbb{R} \oplus \mathbb{R} \oplus \mathbb{R}$. \\
\hline
\end{tabular}

Table 2: Signatures of 3-dimensional unimodular simply-connected Lie groups.

If $\mathcal{F}$ is a Milnor frame for $g$, then $\operatorname{Rc}\left(F_{i}, F_{j}\right)=0$ whenever $i \neq j$. This follows easily from the observation that $\left\langle R\left(F_{k}, F_{i}\right) F_{j}, F_{k}\right\rangle=0$ for all $k$ and any $i \neq j$. In a Milnor frame, therefore, we may regard $g$ and Rc as diagonal matrices and the Ricci flow as a system of three ODE.

Any Milnor frame $\mathcal{F}$ for $g$ determines a 3-dimensional submanifold $\mathcal{D}_{\mathcal{F}} \subset \mathcal{S}_{3}^{+}$consisting of all metrics for which it is a Milnor frame, that is to say, all metrics diagonal with respect to $\mathcal{F}$. This submanifold is not canonically associated to $g$, as the example of Euclidean geometry clearly shows. Nonetheless, it will be useful to begin our analysis of $[g]$ by studying

$$
[g]_{\mathcal{F}} \doteqdot[g] \cap \mathcal{D}_{\mathcal{F}}
$$


2.0.2. The general case. In general, there may not exist a frame $\mathcal{F}$ which is simultaneously a Milnor frame for arbitrary metrics $g, h \in \mathcal{S}_{3}^{+}$. We can, however, use basic linear algebra to overcome this difficulty. We recall that $\mathrm{gl}(\mathfrak{g}) \cong \mathrm{gl}(3, \mathbb{R})$ and begin with some notational conventions.

- If $\beta \in \mathrm{gl}(3, \mathbb{R})$ is regarded as a basis $\beta=\left(F_{1}, F_{2}, F_{3}\right)$, then each $V \in \mathfrak{g}$ defines coordinate functions $V_{\beta}=\left(V_{\beta}^{i}\right)$ by $V=\beta V_{\beta}=F_{i} V_{\beta}^{i}$.

- If $A \in \operatorname{gl}(3, \mathbb{R})$ is regarded as a change of basis by right multiplication $\alpha=\beta A$, then $V_{\alpha}=A^{-1} V_{\beta}$.

- A linear transformation $\Lambda \in \operatorname{gl}(\mathfrak{g})$ defines a matrix $\Lambda_{\beta}$ by $\Lambda V=$ $\beta \Lambda_{\beta} V_{\beta}$; under a change of basis, this becomes $\Lambda_{\alpha}=A^{-1} \Lambda_{\beta} A$.

- A scalar product $h$ defines a matrix $h_{\beta} \in \mathcal{S}_{3}^{+}$by $h(V, W)=V_{\beta}^{\operatorname{tr}} h_{\beta} W_{\beta}$; under a change of basis, this becomes $h_{\alpha}=A^{\text {tr }} h_{\beta} A$.

- We may write the bracket relations in the form $[\beta]=\beta \sigma_{\beta}$, where

$$
[\beta] \doteqdot\left(\left[F_{2}, F_{3}\right],\left[F_{3}, F_{1}\right],\left[F_{1}, F_{2}\right]\right)
$$

and $\sigma_{\beta}$ is the matrix of structure constants

$$
\sigma_{\beta} \doteqdot\left(\begin{array}{ccc}
c_{23}^{1} & c_{31}^{1} & c_{12}^{1} \\
c_{23}^{2} & c_{31}^{2} & c_{12}^{2} \\
c_{23}^{3} & c_{31}^{3} & c_{12}^{3}
\end{array}\right)
$$

Under a change of basis, this becomes $\sigma_{\alpha}=(\operatorname{det} A) \cdot A^{-1} \sigma_{\beta}\left(A^{-1}\right)^{\operatorname{tr}}$, [1].

To complete our analysis, therefore, it is enough to know how to transform Milnor bases in a given geometry:

Algorithm 2.1. Let $g=g(0), h=h(0)$ be two scalar products on a Lie algebra $\mathfrak{g}$. By the Milnor construction, there is a basis $\beta$ such that $g_{\beta}$ and $\sigma_{\beta}$ are diagonal, and a basis $\alpha$ such that $h_{\alpha}$ and $\sigma_{\alpha}$ are diagonal. Compute $A$ depending only on $\mathfrak{g}$ and $g(0), h(0)$ such that $\alpha=\beta A$, and let $B \doteqdot A^{-1}$. Then compute the evolution of $h_{\alpha}$ and compare $g_{\beta}(t)$ with $h_{\beta}(t)$ for any $t \geq 0$ using the identity

$$
h_{\beta}(t)=B^{\operatorname{tr}} h_{\alpha}(t) B
$$




\subsection{The Lie group $\mathbb{R}^{3}$.}

The Ricci flow is trivial and $[g]$ is 0-dimensional for any $g$ in this 6dimensional family of metrics having the geometry of $\mathbb{E}^{3}$.

\section{2. nil-geometry.}

2.2.1. The class $[g]_{\mathcal{F}}$ for a nil-geometry metric $g$. Consider $(\mathcal{G}, g)$, where $\mathcal{G}$ is the Heisenberg group, $\left\{F_{i}\right\}$ is a Milnor frame for $g$ with dual forms $\left\{\omega^{i}\right\}$, and the metric is

$$
g=A \omega^{1} \otimes \omega^{1}+B \omega^{2} \otimes \omega^{2}+C \omega^{3} \otimes \omega^{3} .
$$

We may take $\lambda^{1}=-1$ and $\lambda^{2}=\lambda^{3}=0$. Then the sectional curvatures are

$$
\text { (11) } K\left(F_{2} \wedge F_{3}\right)=-3 \frac{A}{B C}, \quad K\left(F_{3} \wedge F_{1}\right)=\frac{A}{B C}, \quad K\left(F_{1} \wedge F_{2}\right)=\frac{A}{B C},
$$

and the Ricci tensor is

$$
\mathrm{Rc}=2 \frac{A^{2}}{B C} \omega^{1} \otimes \omega^{1}-2 \frac{A}{C} \omega^{2} \otimes \omega^{2}-2 \frac{A}{B} \omega^{3} \otimes \omega^{3} .
$$

Hence if we put $A_{0} \doteqdot A(0), B_{0} \doteqdot B(0)$, and $C_{0} \doteqdot C(0)$, the Ricci flow is equivalent to the system

$$
\frac{d}{d t} A=-4 \frac{A^{2}}{B C}, \quad \frac{d}{d t} B=4 \frac{A}{C}, \quad \frac{d}{d t} C=4 \frac{A}{B} .
$$

Noting that $\frac{d}{d t}(A B)==\frac{d}{d t}(B / C)=0$, we introduce positive constants $\Phi, \Psi$ denoting the conserved quantities

$$
A B=\Phi \doteqdot A_{0} B_{0} \quad \text { and } \quad \frac{B}{C}=\Psi \doteqdot \frac{B_{0}}{C_{0}}
$$

We begin by computing and solving

$$
\frac{d}{d t}\left(\frac{A}{B^{2}}\right)=-12 \Psi\left(\frac{A}{B^{2}}\right)^{2}
$$

to get

$$
\frac{A}{B^{2}}=\frac{C_{0} / B_{0}}{12 t+B_{0} C_{0} / A_{0}} .
$$


This lets us solve

$$
\frac{d}{d t} A=-4 \Psi\left(\frac{A}{B^{2}}\right) A=-\frac{4}{12 t+B_{0} C_{0} / A_{0}} A,
$$

obtaining

$$
\begin{aligned}
& A=A_{0}^{2 / 3} B_{0}^{1 / 3} C_{0}^{1 / 3}\left(12 t+B_{0} C_{0} / A_{0}\right)^{-1 / 3} \\
& B=\frac{\Phi}{A}=A_{0}^{1 / 3} B_{0}^{2 / 3} C_{0}^{-1 / 3}\left(12 t+B_{0} C_{0} / A_{0}\right)^{1 / 3} \\
& C=\frac{B}{\Psi}=A_{0}^{1 / 3} B_{0}^{-1 / 3} C_{0}^{2 / 3}\left(12 t+B_{0} C_{0} / A_{0}\right)^{1 / 3} .
\end{aligned}
$$

Notice that any compact quotient of $\mathcal{G}$ becomes almost flat with $|\mathrm{Rm}| \sim t^{-1}$, $\mathrm{Vol} \sim t^{1 / 6}$, and $\operatorname{diam} \sim t^{1 / 6}$.

Now if

$$
\bar{g}=\bar{A} \omega^{1} \otimes \omega^{1}+\bar{B} \omega^{2} \otimes \omega^{2}+\bar{C} \omega^{3} \otimes \omega^{3}
$$

is another metric in $\mathcal{D}_{\mathcal{F}}$, then

$$
|g-\bar{g}|_{g}^{2}=\left(\frac{A-\bar{A}}{A}\right)^{2}+\left(\frac{B-\bar{B}}{B}\right)^{2}+\left(\frac{C-\bar{C}}{C}\right)^{2},
$$

where

$$
\begin{aligned}
& \frac{A-\bar{A}}{A}=1-\frac{\bar{A}_{0}^{2 / 3} \bar{B}_{0}^{1 / 3} \bar{C}_{0}^{1 / 3}}{A_{0}^{2 / 3} B_{0}^{1 / 3} C_{0}^{1 / 3}}\left(\frac{12 t+B_{0} C_{0} / A_{0}}{12 t+\bar{B}_{0} \bar{C}_{0} / \bar{A}_{0}}\right)^{1 / 3} \rightarrow 1-\frac{\bar{A}_{0}^{2 / 3} \bar{B}_{0}^{1 / 3} \bar{C}_{0}^{1 / 3}}{A_{0}^{2 / 3} B_{0}^{1 / 3} C_{0}^{1 / 3}} \\
& \frac{B-\bar{B}}{B}=1-\frac{\bar{A}_{0}^{1 / 3} \bar{B}_{0}^{2 / 3} \bar{C}_{0}^{-1 / 3}}{A_{0}^{1 / 3} B_{0}^{2 / 3} C_{0}^{-1 / 3}}\left(\frac{12 t+\bar{B}_{0} \bar{C}_{0} / \bar{A}_{0}}{12 t+B_{0} C_{0} / A_{0}}\right)^{1 / 3} \rightarrow 1-\frac{\bar{A}_{0}^{1 / 3} \bar{B}_{0}^{2 / 3} \bar{C}_{0}^{-1 / 3}}{A_{0}^{1 / 3} B_{0}^{2 / 3} C_{0}^{-1 / 3}} \\
& \frac{C-\bar{C}}{C}=1-\frac{\bar{A}_{0}^{1 / 3} \bar{B}_{0}^{-1 / 3} \bar{C}_{0}^{2 / 3}}{A_{0}^{1 / 3} B_{0}^{-1 / 3} C_{0}^{2 / 3}}\left(\frac{12 t+\bar{B}_{0} \bar{C}_{0} / \bar{A}_{0}}{12 t+B_{0} C_{0} / A_{0}}\right)^{1 / 3} \rightarrow 1-\frac{\bar{A}_{0}^{1 / 3} \bar{B}_{0}^{-1 / 3} \bar{C}_{0}^{2 / 3}}{A_{0}^{1 / 3} B_{0}^{-1 / 3} C_{0}^{2 / 3}} .
\end{aligned}
$$

Lemma 2.2. $\bar{g} \in[g]_{\mathcal{F}}$ iff for an arbitrary positive scaling parameter $\lambda$,

$$
\bar{A}_{0}=\frac{A_{0}}{\lambda}, \quad \bar{B}_{0}=\lambda B_{0}, \quad \bar{C}_{0}=\lambda C_{0} .
$$

Proof. Sufficiency is easily checked. To show necessity, choose $\bar{A}_{0}>0$ arbitrarily and define $\lambda$ by

$$
\lambda \doteqdot \frac{A_{0}}{\bar{A}_{0}}
$$


Then $\bar{g} \in[g]_{\mathcal{F}}$ only if

$$
\begin{aligned}
& \bar{A} / A \rightarrow 1 \Rightarrow \bar{B}_{0} \bar{C}_{0}=\lambda^{2} B_{0} C_{0} \\
& \bar{C} / C \rightarrow 1 \Rightarrow B_{0} \bar{C}_{0}^{2}=\lambda \bar{B}_{0} C_{0}^{2} .
\end{aligned}
$$

Solving these equations for $\bar{B}_{0} / B_{0}$ gives

$$
\frac{\lambda^{2} C_{0}}{\bar{C}_{0}}=\frac{\bar{B}_{0}}{B_{0}}=\frac{\bar{C}_{0}^{2}}{\lambda C_{0}^{2}}
$$

which implies $\bar{C}_{0} / C_{0}=\lambda$ and hence $\bar{B}_{0} / B_{0}=\bar{C}_{0}^{2} /\left(\lambda C_{0}^{2}\right)=\lambda$.

The intuition here is that letting $\lambda \rightarrow \infty$ corresponds to letting $t \rightarrow \infty$ in the Ricci flow. In this sense, $\bar{g}$ represents $g$ translated in time. This quasiconvergence behavior is analogous to what was observed for solv-Gowdy metrics in [6], and illustrates a general principle:

Remark 2.3. Let $\left(\mathcal{M}^{n}, g\right)$ be a solution to the Ricci flow for $0 \leq t<\infty$ such that $|\mathrm{Rc}| \rightarrow 0$ uniformly as $t \rightarrow \infty$. For $u>0$, let $\tilde{g}$ denote the solution $\tilde{g}(\cdot, t)=g(\cdot, t+u)$. Then $[\tilde{g}]=[g]$.

Proof. For any $P \in \mathcal{M}^{n}$ and any locally nonzero vector field $V$ near $P$, consider

$$
\frac{\partial}{\partial \tau} \log g(V, V)(P, t+\tau)=-2 \frac{\operatorname{Rc}(V, V)(P, t+\tau)}{g(V, V)(P, t+\tau)} .
$$

For any $\varepsilon>0$, there is $t_{\varepsilon} \geq 0$ independent of $P, V$ such that when $t \geq t_{\varepsilon}$, we have

$$
\left|\frac{\partial}{\partial \tau} \log g(V, V)(P, t+\tau)\right| \leq 2|\mathrm{Rc}|(P, t+\tau) \leq \frac{\varepsilon}{u}
$$

and thus

$$
|\log g(V, V)(P, t+u)-\log g(V, V)(P, t)| \leq \int_{0}^{u} \frac{\varepsilon}{u} d \tau=\varepsilon .
$$

This implies

$$
e^{-\varepsilon} g(V, V)(P, t) \leq \tilde{g}(V, V)(P, t) \leq e^{\varepsilon} g(V, V)(P, t),
$$

whence taking the supremum over all $V$ such that $g(V, V)(P, t)=1$ shows

$$
|g-\tilde{g}|_{g}(P, t) \leq C\left(e^{\varepsilon}-1\right)
$$


where $C$ is a constant depending only on the dimension $n$.

Notice that it is easy to recover $u$ from $\lambda$ using just the scalar curvature. Indeed, setting $\bar{R}(P, t)=\tilde{R}(P, t)$ gives formally

$$
u(\lambda)=\frac{\lambda^{3}-1}{12} \frac{B_{0} C_{0}}{A_{0}} .
$$

The fact that this formula admits solutions $u<0$ illustrates that scaling by $\lambda<1$ amounts to translating a solution backward in time. How far this can be done is of course a function of the initial scalar curvature $R(0)=$ $-2 A_{0} / B_{0} C_{0}$.

2.2.2. The class $[g]$ for a nil-geometry metric $g$. Let $\beta=\left(F_{1}, F_{2}, F_{3}\right)$ and $h_{\beta}=\left(h_{i j}\right)$ be given; we assume $\sigma_{\beta}=\left(\begin{array}{ccc}-2 & & \\ & 0 & \\ & & 0\end{array}\right)$. If $A$ induces the change of basis $\beta \mapsto \alpha=\beta A$ and $B \doteqdot A^{-1}$, then

$$
(\operatorname{det} B) \cdot \sigma_{\alpha}=B \sigma_{\beta} B^{\operatorname{tr}}=-2\left(\begin{array}{ccc}
B_{11}^{2} & B_{11} B_{21} & B_{11} B_{31} \\
B_{11} B_{21} & B_{21}^{2} & B_{21} B_{31} \\
B_{11} B_{31} & B_{21} B_{31} & B_{31}^{2}
\end{array}\right) .
$$

This is diagonal iff $B_{11} B_{21}=B_{11} B_{31}=B_{21} B_{31}=0$. So (up to a permutation) we may suppose that

$$
B=\left(\begin{array}{ccc}
e & f & g \\
& a & b \\
c & d
\end{array}\right) \text { and } A=\frac{1}{(a d-b c) e}\left(\begin{array}{ccc}
a d-b c & c g-d f & b f-a g \\
& d e & -b e \\
& -c e & a e
\end{array}\right) .
$$

Imposing the requirement that $h_{\alpha}$ be diagonal yields the underdetermined system

$$
\begin{aligned}
0 & =(c g-d f) h_{11}+d e h_{12}-c e h_{13} \\
0 & =(b f-a g) h_{11}-b e h_{12}+a e h_{13} \\
0 & =e(2 b d f-a d g-b c g) h_{12}+e(2 a c g-a d f-b c f) h_{13} \\
& +e^{2}(a d+b c) h_{23}+(b f-a g)(c g-d f) h_{11}-b d e^{2} h_{22}-a c e^{2} h_{33} .
\end{aligned}
$$


Recalling that

$$
h_{11}>0 \text { and } h_{11} h_{22}-h_{12}^{2}>0
$$

because $h$ is positive definite, we observe that a solution is given by

$$
B \doteqdot\left(\begin{array}{ccc}
1 & \frac{h_{12}}{h_{11}} & \frac{h_{13}}{h_{11}} \\
& 1 & \frac{h_{11} h_{23}-h_{12} h_{13}}{h_{11} h_{22}-h_{12}^{2}} \\
& 1
\end{array}\right)
$$

Notice that $\operatorname{det} B=1$ and $\sigma_{\alpha}=\sigma_{\beta}$, where

$$
A=\left(\begin{array}{ccc}
1 & -\frac{h_{12}}{h_{11}} & \frac{h_{12} h_{23}-h_{13} h_{22}}{h_{11} h_{22}-h_{12}^{2}} \\
1 & \frac{h_{12} h_{13}-h_{11} h_{23}}{h_{11} h_{22}-h_{12}^{2}} \\
& 1
\end{array}\right)
$$

To simplify the notation, let $H_{n}>0$ denote the determinant of the upper left $n \times n$ submatrix of $h_{\beta}$ :

$$
\begin{aligned}
& H_{1} \doteqdot h_{11} \\
& H_{2} \doteqdot h_{11} h_{22}-h_{12}^{2} \\
& H_{3} \doteqdot h_{11} h_{22} h_{33}+2 h_{12} h_{13} h_{23}-h_{11} h_{23}^{2}-h_{22} h_{13}^{2}-h_{33} h_{12}^{2}
\end{aligned}
$$

Then we can write

(20) $\quad h_{\alpha}=A^{\operatorname{tr}} h_{\beta} A=\left(\begin{array}{ccc}H_{1} & & \\ & H_{2} / H_{1} & \\ & & H_{3} / H_{2}\end{array}\right) \doteqdot\left(\begin{array}{ccc}\mathcal{A}_{0} & & \\ & \mathcal{B}_{0} & \\ & & \mathcal{C}_{0}\end{array}\right)$,

so that the solution $h_{\alpha}(t)$ corresponds to

$$
\begin{aligned}
& \mathcal{A}(t)=H_{1}^{1 / 3} H_{3}^{1 / 3}\left(12 t+H_{3} / H_{1}^{2}\right)^{-1 / 3} \\
& \mathcal{B}(t)=H_{1}^{-1 / 3} H_{2} H_{3}^{-1 / 3}\left(12 t+H_{3} / H_{1}^{2}\right)^{1 / 3} \\
& \mathcal{C}(t)=H_{1}^{2 / 3} H_{2}^{-1} H_{3}^{2 / 3}\left(12 t+H_{3} / H_{1}^{2}\right)^{1 / 3} .
\end{aligned}
$$

This lets us write $h_{\beta}(t)=B^{\operatorname{tr}} h_{\alpha}(t) B$ in the form

$$
h_{\beta}=\left(\begin{array}{ccc}
\mathcal{A} & \frac{h_{12}}{H_{1}} \mathcal{A} & \frac{h_{13}}{H_{1}} \mathcal{A} \\
\frac{h_{12}}{H_{1}} \mathcal{A} & \frac{h_{12}^{2}}{H_{1}^{2}} \mathcal{A}+\mathcal{B} & \frac{h_{12} h_{13}}{H_{1}^{2}} \mathcal{A}+\frac{h_{11} h_{23}-h_{12} h_{13}}{H_{2}} \mathcal{B} \\
\frac{h_{13}}{H_{1}} \mathcal{A} & \frac{h_{12} h_{13}}{H_{1}^{2}} \mathcal{A}+\frac{h_{11} h_{23}-h_{12} h_{13}}{H_{2}} \mathcal{B} & \frac{h_{13}^{2}}{H_{1}^{2}} \mathcal{A}+\frac{\left(h_{11} h_{23}-h_{12} h_{13}\right)^{2}}{H_{2}^{2}} \mathcal{B}+\mathcal{C}
\end{array}\right) .
$$


Theorem 2.4. The quasi-convergence class $[g]$ of an arbitrary nil-metric $g$ is exactly a 3-parameter family.

Proof. Without loss of generality, we may assume that with respect to a Milnor frame, $g$ is represented by a diagonal matrix corresponding to the initial data $\left(A_{0}, B_{0}, C_{0}\right)$. Then $h \in[g]$ if and only if every term converges to 0 in the sum

$$
\begin{aligned}
|h-g|_{g}^{2}= & \frac{(\mathcal{A}-A)^{2}}{A^{2}}+\frac{\left(\frac{h_{12}^{2}}{H_{1}^{2}} \mathcal{A}+\mathcal{B}-B\right)^{2}}{B^{2}} \\
& +\frac{\left(\frac{h_{13}^{2}}{H_{1}^{2}} \mathcal{A}+\frac{\left(h_{11} h_{23}-h_{12} h_{13}\right)^{2}}{H_{2}^{2}} \mathcal{B}+\mathcal{C}-C\right)^{2}}{C^{2}}+2 \frac{\left(\frac{h_{12}}{H_{1}} \mathcal{A}\right)^{2}}{A B} \\
& +2 \frac{\left(\frac{h_{13}}{H_{1}} \mathcal{A}\right)^{2}}{A C}+2 \frac{\left(\frac{h_{12} h_{13}}{H_{1}^{2}} \mathcal{A}+\frac{h_{11} h_{23}-h_{12} h_{13}}{H_{2}} \mathcal{B}\right)^{2}}{B C}
\end{aligned}
$$

To see that $[g]$ is at least 3 -dimensional, let $\lambda>0$ and $\mu, \nu \in \mathbb{R}$ be given and put

$$
h_{11}=A_{0} / \lambda, \quad h_{12}=\mu, \quad h_{13}=\nu
$$

If

$$
h_{23}=\frac{h_{12} h_{13}}{h_{11}}, \quad h_{22}=\lambda B_{0}+\frac{h_{12}^{2}}{h_{11}}, \quad h_{33}=\lambda C_{0}+\frac{h_{22} h_{13}^{2}-h_{11} h_{23}^{2}}{A_{0} B_{0}},
$$

then $H_{1}=A_{0} / \lambda, H_{2}=A_{0} B_{0}$, and $H_{3}=\lambda A_{0} B_{0} C_{0}$. Therefore $h(0)$ is positive definite, and $\mathcal{A} / A \rightarrow 1, \mathcal{B} / B \rightarrow 1$, and $\mathcal{C} / C \rightarrow 1$. Because $h_{11} h_{23}=$ $h_{12} h_{13}$, it follows easily that $|h-g|_{g} \rightarrow 0$.

To see that $[g]$ is at most 3-dimensional, suppose $h \in[g]$. Then clearly $h_{11} h_{23}=h_{12} h_{13}$, and we must have

$$
1=\lim _{t \rightarrow \infty} \frac{\mathcal{A}}{A}=\frac{H_{1}^{1 / 3} H_{3}^{1 / 3}}{A_{0}^{2 / 3} B_{0}^{1 / 3} C_{0}^{1 / 3}}=\frac{\mathcal{A}_{0}^{2 / 3} \mathcal{B}_{0}^{1 / 3} \mathcal{C}_{0}^{1 / 3}}{A_{0}^{2 / 3} B_{0}^{1 / 3} C_{0}^{1 / 3}}
$$

and

$$
1=\lim _{t \rightarrow \infty} \frac{\mathcal{B}}{B}=\frac{H_{1}^{-1 / 3} H_{2} H_{3}^{-1 / 3}}{A_{0}^{1 / 3} B_{0}^{2 / 3} C_{0}^{-1 / 3}}=\frac{\mathcal{A}_{0}^{1 / 3} \mathcal{B}_{0}^{2 / 3} \mathcal{C}_{0}^{-1 / 3}}{A_{0}^{1 / 3} B_{0}^{2 / 3} C_{0}^{-1 / 3}}
$$

and

$$
1=\lim _{t \rightarrow \infty} \frac{\mathcal{C}}{C}=\frac{H_{1}^{2 / 3} H_{2}^{-1} H_{3}^{2 / 3}}{A_{0}^{1 / 3} B_{0}^{-1 / 3} C_{0}^{2 / 3}}=\frac{\mathcal{A}_{0}^{1 / 3} \mathcal{B}_{0}^{-1 / 3} \mathcal{C}_{0}^{2 / 3}}{A_{0}^{1 / 3} B_{0}^{-1 / 3} C_{0}^{2 / 3}}
$$


As in the diagonal case, it follows that $\mathcal{A}_{0} \mathcal{B}_{0}=A_{0} B_{0}$ and $\mathcal{A}_{0} \mathcal{C}_{0}=A_{0} C_{0}$. Thus if $h_{11}$ and any two of $h_{12}, h_{13}, h_{23}$ are prescribed, the remaining $h_{i j}$ are determined.

\subsection{The Lie group Isom( $\widetilde{\left.\mathbb{E}^{2}\right)}$.}

2.3.1. The class $[g]_{\mathcal{F}}$ for an $\operatorname{Isom}\left(\mathbb{E}^{2}\right)$-metric $g$. Consider $(\mathcal{G}, g)$, where $\mathcal{G}=\widetilde{\operatorname{Isom}\left(\mathbb{E}^{2}\right)},\left\{F_{i}\right\}$ is a Milnor frame with dual forms $\left\{\omega^{i}\right\}$, and the metric once again is written as

$$
g=A \omega^{1} \otimes \omega^{1}+B \omega^{2} \otimes \omega^{2}+C \omega^{3} \otimes \omega^{3} .
$$

We may take $\lambda^{1}=\lambda^{2}=-1$ and $\lambda^{3}=0$. Then the sectional curvatures are

$$
\begin{aligned}
& K\left(F_{2} \wedge F_{3}\right)=\frac{(A+B)^{2}-4 A^{2}}{A B C} \\
& K\left(F_{3} \wedge F_{1}\right)=\frac{(A+B)^{2}-4 B^{2}}{A B C} \\
& K\left(F_{1} \wedge F_{2}\right)=\frac{(A-B)^{2}}{A B C},
\end{aligned}
$$

and the Ricci flow is equivalent to the system

$$
\frac{d}{d t} A=4 \frac{B^{2}-A^{2}}{B C}, \quad \frac{d}{d t} B=4 \frac{A^{2}-B^{2}}{A C}, \quad \frac{d}{d t} C=4 \frac{(A-B)^{2}}{A B},
$$

with $A_{0} \doteqdot A(0), B_{0} \doteqdot B(0)$, and $C_{0} \doteqdot C(0)$. Clearly, the geometry is flat (and the Ricci flow is trivial) iff $A=B$. Noting that $\frac{d}{d t}(A B)=$ $\frac{d}{d t}(C(A+B))=0$, we denote these conserved quantities by

$$
A B=\Phi \doteqdot A_{0} B_{0} \quad \text { and } \quad C(A+B)=\Psi \doteqdot C_{0}\left(A_{0}+B_{0}\right) \text {. }
$$

We can get enough qualitative information to describe the class $[g]_{\mathcal{F}}$ by setting $\rho \doteqdot B / A$ and considering the simplified system

$$
\frac{d}{d t} \rho=8 \frac{1-\rho^{2}}{C}, \quad \frac{d}{d t} C=4 \frac{(1-\rho)^{2}}{\rho},
$$

where $\rho_{0} \doteqdot \rho(0)$ and $C_{0}$ are positive.

Lemma 2.5. Ast $\rightarrow \infty, \rho$ converges to 1 and $C$ converges to some $C_{\infty}>0$. 
Proof. Since every point on the ray $\rho=1, C>0$ is a fixed point, we may assume that $\rho_{0} \neq 1$, hence that $\rho \neq 1$ for all time by uniqueness of solutions to ODE. Because $d \rho / d t>0$ if $0<\rho<1$ and $d \rho / d t<0$ if $1<\rho$, it follows that $\rho$ is bounded and monotone, hence approaches a limit $\rho_{\infty}$ satisfying either $\rho_{0}<\rho_{\infty} \leq 1$ or $1 \leq \rho_{\infty}<\rho_{0}$. Now since $\rho$ is strictly monotone, we may regard $C$ as a function of $\rho$; then we have

$$
\frac{d}{d \rho} \log C=\frac{1}{2 \rho} \frac{1-\rho}{1+\rho}
$$

which lets us conclude that $\lim _{t \rightarrow \infty} C(t)$ exists, because

$$
\log \frac{C(\rho(t))}{C_{0}}=\int_{\rho_{0}}^{\rho(t)} \frac{1}{2 \rho} \frac{1-\rho}{1+\rho} d \rho \rightarrow \log \frac{\sqrt{\rho_{\infty}}}{1+\rho_{\infty}}-\log \frac{\sqrt{\rho_{0}}}{1+\rho_{0}} \quad \text { as } \quad t \rightarrow \infty .
$$

It follows that $\rho_{\infty}=1$ : if not, there would be some $\varepsilon>0$ such that $d C / d t \geq$ $\varepsilon$ for all time, an evident contradiction.

This observation proves that

$$
\lim _{t \rightarrow \infty} A=\lim _{t \rightarrow \infty} B=\sqrt{\Phi}
$$

and

$$
\lim _{t \rightarrow \infty} C=\frac{C_{0}}{2}\left(\sqrt{\frac{A_{0}}{B_{0}}}+\sqrt{\frac{B_{0}}{A_{0}}}\right) .
$$

Corollary 2.6. If $g$ is an $\operatorname{Isom}\left(\mathbb{E}^{2}\right)$ metric, then $[g]_{\mathcal{F}}$ is exactly a 1parameter family.

Proof. Let $\left(A_{0}, B_{0}, C_{0}\right)$ be given and let $\bar{g}$ correspond to the initial data $\left(\bar{A}_{0}, \bar{B}_{0}, \bar{C}_{0}\right)$, noting that $\bar{g} \in[g]_{\mathcal{F}}$ iff $\bar{A} / A, \bar{B} / B$, and $\bar{C} / C$ all converge to 1. Choose $\bar{A}_{0}>0$ arbitrarily. Then $\bar{A} / A \rightarrow 1$ and $\bar{B} / B \rightarrow 1$ iff

$$
\bar{B}_{0}=\frac{\Phi}{\bar{A}_{0}} \text {. }
$$

And $\bar{C} / C \rightarrow 1$ iff

$$
\bar{C}_{0}=\frac{\sqrt{A_{0} / B_{0}}+\sqrt{B_{0} / A_{0}}}{\sqrt{\bar{A}_{0} / \bar{B}_{0}}+\sqrt{\bar{B}_{0} / \bar{A}_{0}}} C=\frac{A_{0} / \sqrt{\Phi}+B_{0} / \sqrt{\Phi}}{\bar{A}_{0} / \sqrt{\Phi}+\bar{B}_{0} / \sqrt{\Phi}} C=\frac{A_{0}+B_{0}}{\bar{A}_{0}+\Phi / \bar{A}_{0}} C .
$$


In spite of the existence of nontrivial quasi-convergence classes, it is worth noting that the Ricci flow of Isom $\left(\mathbb{E}^{2}\right)$ metrics actually does converge: the metric becomes asymptotically flat, and the volume of any compact quotient increases monotonically to a finite limit.

2.3.2. The class $[g]$ for an Isom $\left(\mathbb{E}^{2}\right)$-metric $g$. Let $\beta=\left(F_{1}, F_{2}, F_{3}\right)$ and $h_{\beta}=\left(h_{i j}\right)$ be given; we assume $\sigma_{\beta}=\left(\begin{array}{ccc}-2 & & \\ & -2 & \\ & & 0\end{array}\right)$. If $A$ induces the change of basis $\beta \mapsto \alpha=\beta A$ and $B \doteqdot A^{-1}$, then

$$
\frac{\operatorname{det} B}{-2} \sigma_{\alpha}=\left(\begin{array}{ccc}
B_{11}^{2}+B_{12}^{2} & B_{11} B_{21}+B_{12} B_{22} & B_{11} B_{31}+B_{12} B_{32} \\
B_{11} B_{21}+B_{12} B_{22} & B_{21}^{2}+B_{22}^{2} & B_{21} B_{31}+B_{22} B_{32} \\
B_{11} B_{31}+B_{12} B_{32} & B_{21} B_{31}+B_{22} B_{32} & B_{31}^{2}+B_{32}^{2}
\end{array}\right) .
$$

So we seek matrices of the form

$$
B=\left(\begin{array}{ccc}
a & b & c \\
-b & a & d \\
& & 1
\end{array}\right) \text { and } A=\frac{1}{\left(a^{2}+b^{2}\right)}\left(\begin{array}{ccc}
a & -b & b d-a c \\
b & a & -a d-b c \\
& & a^{2}+b^{2}
\end{array}\right) \text {. }
$$

Asking that $h_{\alpha}$ be diagonal gives the system

$$
\begin{aligned}
0= & a\left(a h_{12}-b h_{11}\right)+b\left(a h_{22}-b h_{12}\right) \\
0= & \left(a h_{11}+b h_{12}\right)(b d-a c)-\left(a h_{12}+b h_{22}\right)(a d+b c) \\
& +\left(a h_{13}+b h_{23}\right)\left(a^{2}+b^{2}\right) \\
0= & \left(a h_{12}-b h_{11}\right)(b d-a c)-\left(a h_{22}-b h_{12}\right)(a d+b c) \\
& +\left(a h_{23}-b h_{13}\right)\left(a^{2}+b^{2}\right) .
\end{aligned}
$$

Define

$$
\omega \doteqdot\left\{\begin{array}{cc}
1 & \text { if } h_{12}=0 \\
\frac{h_{11}-h_{22}+\sqrt{\left(h_{11}-h_{22}\right)^{2}+4 h_{12}^{2}}}{2} & \text { if } h_{12} \neq 0,
\end{array}\right.
$$

noting that we always have

$$
h_{12}\left(\omega^{2}+\left(h_{22}-h_{11}\right) \omega-h_{12}^{2}\right)=0 .
$$


(The fact that $\omega$ may not be a continuous function of $h_{12}$ will not be an issue.) In case $h_{12} \neq 0$, let

$$
\hat{A} \doteqdot\left(\begin{array}{ccc}
\omega & -h_{12} & \\
h_{12} & \omega & \\
& & 1
\end{array}\right)
$$

and notice that the change of basis $\beta \mapsto \beta \hat{A}$ transforms $h_{\beta}=\left(h_{i j}\right)$ into $\left(\hat{h}_{i j}\right)$, where

$$
\begin{aligned}
& \hat{h}_{11}=\omega^{2} h_{11}+2 h_{12}^{2} \omega+h_{12}^{2} h_{22}>0 \\
& \hat{h}_{22}=\omega^{2} h_{22}-2 h_{12}^{2} \omega+h_{12}^{2} h_{11}>0 \\
& \hat{h}_{13}=\omega h_{13}+h_{12} h_{23} \\
& \hat{h}_{23}=\omega h_{23}-h_{12} h_{13} .
\end{aligned}
$$

So in any case we can define

$$
\begin{aligned}
& \phi \doteqdot \frac{\omega h_{13}+h_{12} h_{23}}{\omega^{2} h_{11}+2 h_{12}^{2} \omega+h_{12}^{2} h_{22}} \\
& \psi \doteqdot \frac{\omega h_{23}-h_{12} h_{13}}{\omega^{2} h_{22}-2 h_{12}^{2} \omega+h_{12}^{2} h_{11}}
\end{aligned}
$$

and obtain a suitable change of basis by setting

$$
B \doteqdot\left(\begin{array}{ccc}
\omega & h_{12} & \phi \\
-h_{12} & \omega & \psi \\
& & 1
\end{array}\right)
$$

Indeed, we have $\sigma_{\alpha}=\sigma_{\beta}$ and

$$
A=\frac{1}{\omega^{2}+h_{12}^{2}}\left(\begin{array}{ccc}
\omega & -h_{12} & h_{12} \psi-\omega \phi \\
h_{12} & \omega & -h_{12} \phi-\omega \psi \\
& & \omega^{2}+h_{12}^{2}
\end{array}\right)
$$

and find there are $\mathcal{A}_{0}, \mathcal{B}_{0}, \mathcal{C}_{0}>0$ such that $h_{\alpha}=A^{\operatorname{tr}} h_{\beta} A=$ $\left(\mathcal{A}_{0}\right.$

$\left.\begin{array}{cc} & \\ \mathcal{B}_{0} & \\ & \mathcal{C}_{0}\end{array}\right)$ 
general case.) We compute that $h_{\beta}(t)=B^{\operatorname{tr}} h_{\alpha}(t) B$ is given by

$$
h_{\beta}=\left(\begin{array}{ccc}
\omega^{2} \mathcal{A}+h_{12}^{2} \mathcal{B} & \omega h_{12}(\mathcal{A}-\mathcal{B}) & \omega \phi \mathcal{A}-h_{12} \psi \mathcal{B} \\
\omega h_{12}(\mathcal{A}-\mathcal{B}) & h_{12}^{2} \mathcal{A}+\omega^{2} \mathcal{B} & h_{12} \phi \mathcal{A}+\omega \psi \mathcal{B} \\
\omega \phi \mathcal{A}-h_{12} \psi \mathcal{B} & h_{12} \phi \mathcal{A}+\omega \psi \mathcal{B} & \phi^{2} \mathcal{A}+\psi^{2} \mathcal{B}+\mathcal{C}
\end{array}\right)
$$

Theorem 2.7. The quasi-convergence class $[g]$ of an arbitrary $\operatorname{Isom}\left(\mathbb{E}^{2}\right)$ metric $g$ is exactly 2-dimensional.

Proof. Without loss of generality, we may assume that with respect to a frame field for which all structure constants vanish except $c_{23}^{1}=c_{31}^{2}=$ $-2, g$ is represented by a diagonal matrix corresponding to the initial data $\left(A_{0}, B_{0}, C_{0}\right)$. Then

$$
\begin{aligned}
|h-g|_{g}^{2}= & \frac{\left(\omega^{2} \mathcal{A}+h_{12}^{2} \mathcal{B}-A\right)^{2}}{A^{2}}+\frac{\left(h_{12}^{2} \mathcal{A}+\omega^{2} \mathcal{B}-B\right)^{2}}{B^{2}} \\
& +\frac{\left(\phi^{2} \mathcal{A}+\psi^{2} \mathcal{B}+\mathcal{C}-C\right)^{2}}{C^{2}} \\
& +2 \frac{\left(\omega h_{12}(\mathcal{A}-\mathcal{B})\right)^{2}}{A B}+2 \frac{\left(\omega \phi \mathcal{A}-h_{12} \psi \mathcal{B}\right)^{2}}{A C}+2 \frac{\left(h_{12} \phi \mathcal{A}+\omega \psi \mathcal{B}\right)^{2}}{B C} .
\end{aligned}
$$

To see that $[g]$ is at least 2-dimensional, let $h_{11}>0$ and $h_{12}$ be arbitrary and take

$$
h_{22}=\frac{A_{0} B_{0}+h_{12}^{2}}{h_{11}}, \quad h_{13}=0, \quad h_{23}=0,
$$

noting that $\phi=\psi=0$. Then compute

$$
\mathcal{A}_{0}=\frac{\omega^{2} h_{11}+h_{12}^{2}\left(h_{22}+2 \omega\right)}{\left(\omega^{2}+h_{12}^{2}\right)^{2}}, \quad \mathcal{B}_{0}=\frac{\omega^{2} h_{22}+h_{12}^{2}\left(h_{11}-2 \omega\right)}{\left(\omega^{2}+h_{12}^{2}\right)^{2}}, \quad \mathcal{C}_{0}=h_{33}
$$

and set $h_{33}=C_{0}\left(A_{0}+B_{0}\right) /\left(\mathcal{A}_{0}+\mathcal{B}_{0}\right)$. Since $\lim _{t \rightarrow \infty} \mathcal{A}=\lim _{t \rightarrow \infty} \mathcal{B}=$ $\sqrt{\mathcal{A}_{0} \mathcal{B}_{0}}$ and $\lim _{t \rightarrow \infty} \mathcal{C}=\frac{\mathcal{C}_{0}}{2}\left(\sqrt{\frac{\mathcal{A}_{0}}{\mathcal{B}_{0}}}+\sqrt{\frac{\mathcal{B}_{0}}{\mathcal{A}_{0}}}\right)$, it follows from our analysis of the diagonal case that $h \in[g]$ iff

$$
\left(\omega^{2}+h_{12}^{2}\right)^{2} \mathcal{A}_{0} \mathcal{B}_{0}=A_{0} B_{0}
$$


But since $h_{11} h_{22}=A_{0} B_{0}+h_{12}^{2}$, expanding and collecting terms shows that

$$
\begin{aligned}
\left(\omega^{2}+h_{12}^{2}\right)^{2} \mathcal{A}_{0} \mathcal{B}_{0} & =\frac{\left(\omega^{2} h_{11}+h_{12}^{2}\left(h_{22}+2 \omega\right)\right)\left(\omega^{2} h_{22}+h_{12}^{2}\left(h_{11}-2 \omega\right)\right)}{\left(\omega^{2}+h_{12}^{2}\right)^{2}} \\
& =A_{0} B_{0}+\frac{h_{12}^{2}\left(\omega^{2}+\left(h_{22}-h_{11}\right) \omega-h_{12}^{2}\right)^{2}}{\left(\omega^{2}+h_{12}^{2}\right)^{2}}=A_{0} B_{0} .
\end{aligned}
$$

To see that $[g]$ is at most 2-dimensional, we first assert that $h \in[g]$ only if $h_{13}=h_{23}=0$. Indeed, if $h \in[g]$, then $\phi, \psi$ satisfy the system

$$
0=\omega \phi-h_{12} \psi, \quad 0=h_{12} \phi+\omega \psi .
$$

Because this has only the trivial solution $h_{13}=h_{23}=0$ when $h_{12}=0$, we may assume $h_{12} \neq 0$. Then since

$$
0=\omega\left(h_{12} \phi+\omega \psi\right)-h_{12}\left(\omega \phi-h_{12} \psi\right)=\left(\omega^{2}+h_{12}^{2}\right) \psi,
$$

we have $\psi=0$ and thus $\phi=0$. It follows that

$$
h_{13}=\frac{\omega}{h_{12}} h_{23}=-\frac{\omega}{h_{12}} \frac{\omega}{h_{12}} h_{13},
$$

whence we get $h_{13}=0$ and then $h_{23}=0$, which proves the assertion. Then the computations above show that $h_{33}$ is determined by $h_{11}, h_{22}, h_{12}$, and the conservation law $\mathcal{C}_{0}\left(\mathcal{A}_{0}+\mathcal{B}_{0}\right)=C_{0}\left(A_{0}+B_{0}\right)$. Now if the remaining relation $\left(\omega^{2}+h_{12}^{2}\right)^{2} \mathcal{A}_{0} \mathcal{B}_{0}=A_{0} B_{0}$ imposed on $h_{11}, h_{22}, h_{12}$ were vacuous, the restriction $h_{12}=0$ would make the diagonal equivalence class $[g]_{\mathcal{F}}$ a 2-parameter family. Since this is false, $[g]$ is at most 2-dimensional.

\section{4. solv-geometry.}

2.4.1. The class $[g]_{\mathcal{F}}$ for a solv-geometry metric $g$. Consider $(\mathcal{G}, g)$, where $\mathcal{G}=\widetilde{\operatorname{Isom}\left(\mathbb{E}_{1}^{1}\right)}$ (the group of rigid motions of Minkowski 2-space), $\left\{F_{i}\right\}$ is a Milnor frame with dual forms $\left\{\omega^{i}\right\}$, and the metric is

$$
g=A \omega^{1} \otimes \omega^{1}+B \omega^{2} \otimes \omega^{2}+C \omega^{3} \otimes \omega^{3},
$$

with $\lambda^{1}=-1, \lambda^{2}=0$, and $\lambda^{3}=1$. The sectional curvatures are

$$
\begin{aligned}
& K\left(F_{2} \wedge F_{3}\right)=\frac{(A-C)^{2}-4 A^{2}}{A B C} \\
& K\left(F_{3} \wedge F_{1}\right)=\frac{(A+C)^{2}}{A B C} \\
& K\left(F_{1} \wedge F_{2}\right)=\frac{(A-C)^{2}-4 C^{2}}{A B C} .
\end{aligned}
$$


If $A_{0} \doteqdot A(0), B_{0} \doteqdot B(0)$, and $C_{0} \doteqdot C(0)$, then the Ricci flow is equivalent to the system

$$
\frac{d}{d t} A=4 \frac{C^{2}-A^{2}}{B C}, \quad \frac{d}{d t} B=4 \frac{(A+C)^{2}}{A C}, \quad \frac{d}{d t} C=4 \frac{A^{2}-C^{2}}{A B} .
$$

Observing that $\frac{d}{d t}(A C)=\frac{d}{d t}(B(C-A))=0$, we denote these conserved quantities by

$$
A C=\Phi \doteqdot A_{0} C_{0} \quad \text { and } \quad B(C-A)=\Psi \doteqdot B_{0}\left(C_{0}-A_{0}\right) .
$$

Now put $\rho \doteqdot A / C$ and consider the simplified system

$$
\frac{d}{d t} \rho=8 \frac{1-\rho^{2}}{B}, \quad \frac{d}{d t} B=8+4 \frac{1+\rho^{2}}{\rho} \geq 16 .
$$

If $A_{0}=C_{0}$, then $\Psi=0, \rho \equiv 1$, and $B$ grows linearly with time. If not, then by arguing as in the previous section, we see that $\rho$ is strictly monotone and approaches a limit $\rho_{\infty}$ satisfying either $\rho_{0}<\rho_{\infty} \leq 1$ or $1 \leq \rho_{\infty}<\rho_{0}$. Since

$$
\frac{d}{d \rho} \log B=\frac{1}{2 \rho} \frac{1+\rho}{1-\rho}
$$

the condition $\rho_{\infty} \neq 1$ is incompatible with the observation that $B \rightarrow+\infty$. It follows easily that $A, C \rightarrow \sqrt{\Phi}$.

Proposition 2.8. $[g]_{\mathcal{F}}$ is exactly a 2-parameter family.

Proof. Let $\bar{g} \in \mathcal{D}_{\mathcal{F}}$ correspond to the initial data $\left(\bar{A}_{0}, \bar{B}_{0}, \bar{C}_{0}\right)$. Choose $\bar{A}_{0}$ and $\bar{B}_{0}$ arbitrarily. Then clearly, $\bar{A} / A \rightarrow 1$ and $\bar{C} / C \rightarrow 1$ iff $\bar{\Phi}=\Phi$, namely iff

$$
\bar{C}_{0}=\frac{A_{0} C_{0}}{\bar{A}_{0}} .
$$

Then de l'Hôpital shows that

$$
\lim _{t \rightarrow \infty} \frac{\bar{B}}{\bar{B}}=\lim _{t \rightarrow \infty} \frac{A C(\bar{A}+\bar{C})^{2}}{\bar{A} \bar{C}(A+C)^{2}}=1 .
$$

It is perhaps worth relating this result to that obtained in [6]. 
Remark 2.9. If $g$ is a solv-Gowdy metric, the 1-parameter family of locally homogeneous solv-Gowdy metrics in its quasi-convergence equivalence class corresponds to fixing $\bar{A}_{0}=\sqrt{\Phi}$ and letting $\bar{B}_{0}$ vary.

Proof. Suppose $g$ is a homogeneous solv-Gowdy metric, written as

$$
g=e^{2 L} d \theta \otimes d \theta+e^{F+W} d x \otimes d x+e^{F-W} d y \otimes d y
$$

in coordinates $(\theta, x, y)$ on $\mathcal{G} \approx \mathbb{R}^{3}$. A Milnor frame for $g$ is then

$$
\begin{aligned}
& F_{1}=e^{-W / 2} \frac{\partial}{\partial x}-e^{W / 2} \frac{\partial}{\partial y} \\
& F_{2}=\frac{4}{Z} e^{-L} \frac{\partial}{\partial \theta} \\
& F_{3}=e^{-W / 2} \frac{\partial}{\partial x}+e^{W / 2} \frac{\partial}{\partial y}
\end{aligned}
$$

where $Z=\partial W / \partial s$ is positive and constant in space. Indeed, it is easy to check that $\left[F_{1}, F_{2}\right]=2 F_{3},\left[F_{2}, F_{3}\right]=-2 F_{1},\left[F_{3}, F_{1}\right]=0$, and

$$
g=2 e^{F} \omega^{1} \otimes \omega^{1}+\frac{16}{Z^{2}} \omega^{2} \otimes \omega^{2}+2 e^{F} \omega^{3} \otimes \omega^{3} .
$$

So for homogeneous solv-Gowdy metrics, we have $A=C$ and thus $\Phi=4 e^{2 F}$ and $\Psi=0$.

2.4.2. The class $[g]$ for a solv-geometry metric $g$. Let $\beta=\left(F_{1}, F_{2}, F_{3}\right)$ and $h_{\beta}=\left(h_{i j}\right)$ be given; we assume $\sigma_{\beta}=\left(\begin{array}{ccc}-2 & & \\ & 0 & \\ & & 2\end{array}\right)$. If $A$ induces the change of basis $\beta \mapsto \alpha=\beta A$ and $B \doteqdot A^{-1}$, then

$$
\frac{\operatorname{det} B}{2} \sigma_{\alpha}=\left(\begin{array}{ccc}
B_{13}^{2}-B_{11}^{2} & B_{13} B_{23}-B_{11} B_{21} & B_{13} B_{33}-B_{11} B_{31} \\
B_{13} B_{23}-B_{11} B_{21} & B_{23}^{2}-B_{21}^{2} & B_{23} B_{33}-B_{21} B_{31} \\
B_{13} B_{33}-B_{11} B_{31} & B_{23} B_{33}-B_{21} B_{31} & B_{33}^{2}-B_{31}^{2}
\end{array}\right) .
$$

So we seek matrices of the form

$$
B=\left(\begin{array}{ccc}
a & b & c \\
& d & \\
e & f & g
\end{array}\right) \text { and } A=\frac{1}{(a g-c e) d}\left(\begin{array}{ccc}
d g & c f-b g & -c d \\
& a g-c e & \\
-d e & b e-a f & a d
\end{array}\right),
$$


subject to the constraints $d \neq 0$ and $a g \neq c e$ but $a e=c g$. Imposing the requirement that $h_{\alpha}$ be diagonal yields the system

$$
\begin{aligned}
0= & a e-c g \\
0= & \left(g h_{11}-e h_{13}\right)(c f-b g)+\left(g h_{12}-e h_{23}\right)(a g-c e) \\
& +\left(g h_{13}-e h_{33}\right)(b e-a f) \\
0= & \left(a h_{13}-c h_{11}\right) g+\left(c h_{13}-a h_{33}\right) e \\
0= & \left(a h_{13}-c h_{11}\right)(c f-b g)+\left(a h_{23}-c h_{12}\right)(a g-c e) \\
& +\left(a h_{33}-c h_{13}\right)(b e-a f) .
\end{aligned}
$$

Observe that

$$
0<h\left(F_{3}-F_{1}, F_{3}-F_{1}\right)=h_{11}+h_{33}-2 h_{13}
$$

and define

$$
\omega \doteqdot \frac{h_{11}+h_{33}+\sqrt{\left(h_{11}+h_{33}\right)^{2}-4 h_{13}^{2}}}{2},
$$

noting that $\omega$ solves $\omega^{2}-\left(h_{11}+h_{33}\right) \omega+h_{13}^{2}=0$ and obeys the inequality

$$
\Delta \doteqdot \omega^{2}-h_{13}^{2}>0 \text {. }
$$

If

$$
\hat{A} \doteqdot\left(\begin{array}{ccc}
\omega & & h_{13} \\
& 1 & \\
h_{13} & & \omega
\end{array}\right)
$$

then the change of basis $\beta \mapsto \beta \hat{A}$ transforms $h_{\beta}=\left(h_{i j}\right)$ into $\left(\hat{h}_{i j}\right)$, where

$$
\begin{aligned}
& \hat{h}_{11}=\omega^{2} h_{11}-2 \omega h_{13}^{2}+h_{13}^{2} h_{33}>0 \\
& \hat{h}_{33}=\omega^{2} h_{33}-2 \omega h_{13}^{2}+h_{13}^{2} h_{11}>0 \\
& \hat{h}_{12}=\left(\omega h_{12}-h_{13} h_{23}\right) \Delta \\
& \hat{h}_{23}=\left(\omega h_{23}-h_{13} h_{12}\right) \Delta .
\end{aligned}
$$

Hence we may define

$$
\begin{aligned}
& \phi \doteqdot \frac{\hat{h}_{12}}{\hat{h}_{11}}=\frac{\left(\omega h_{12}-h_{13} h_{23}\right) \Delta}{\omega^{2} h_{11}-2 \omega h_{13}^{2}+h_{13}^{2} h_{33}} \\
& \psi \doteqdot \frac{\hat{h}_{23}}{\hat{h}_{33}}=\frac{\left(\omega h_{23}-h_{13} h_{12}\right) \Delta}{\omega^{2} h_{33}-2 \omega h_{13}^{2}+h_{13}^{2} h_{11}} .
\end{aligned}
$$


Then a straightforward computation shows that taking

$$
B \doteqdot\left(\begin{array}{ccc}
\omega & \phi & h_{13} \\
& 1 & \\
h_{13} & \psi & \omega
\end{array}\right)
$$

gives a suitable change of basis

$$
A=\frac{1}{\Delta}\left(\begin{array}{ccc}
\omega & \psi h_{13}-\omega \phi & -h_{13} \\
& \omega^{2}-h_{13}^{2} & \\
-h_{13} & \phi h_{13}-\omega \psi & \omega
\end{array}\right)
$$

Indeed, $\sigma_{\alpha}=\sigma_{\beta}$ and $h_{\alpha}=A^{\text {tr }} h_{\beta} A=\left(\begin{array}{ccc}\mathcal{A}_{0} & & \\ & \mathcal{B}_{0} & \\ & & \mathcal{C}_{0}\end{array}\right)$, where

$$
\begin{aligned}
& \mathcal{A}_{0} \doteqdot \frac{\omega\left(h_{11}^{2}-2 h_{13}^{2}+h_{11} h_{33}\right)+h_{13}^{2}\left(h_{33}-h_{11}\right)}{\Delta^{2}} \\
& \mathcal{C}_{0} \doteqdot \frac{\omega\left(h_{33}^{2}-2 h_{13}^{2}+h_{11} h_{33}\right)+h_{13}^{2}\left(h_{11}-h_{33}\right)}{\Delta^{2}} .
\end{aligned}
$$

It is not useful to compute $\mathcal{B}_{0}$ explicitly. Indeed, our analysis of the diagonal case proves that $\mathcal{A}(t)$ and $\mathcal{C}(t)$ converge to $\sqrt{\mathcal{A}_{0} \mathcal{C}_{0}}>0$; in the special case $h_{13}=0$, we have $\left.\sqrt{\mathcal{A}_{0} \mathcal{C}_{0}}\right|_{h_{13}=0}=\sqrt{h_{11} h_{33}} / \omega^{2}$. Since $h_{\beta}(t)=B^{\operatorname{tr}} h_{\alpha}(t) B$ is

$$
h_{\beta}=\left(\begin{array}{ccc}
\omega^{2} \mathcal{A}+h_{13}^{2} \mathcal{C} & \omega \phi \mathcal{A}+h_{13} \psi \mathcal{C} & \omega h_{13}(\mathcal{A}+\mathcal{C}) \\
\omega \phi \mathcal{A}+h_{13} \psi \mathcal{C} & \phi^{2} \mathcal{A}+\mathcal{B}+\psi^{2} \mathcal{C} & h_{13} \phi \mathcal{A}+\omega \psi \mathcal{C} \\
\omega h_{13}(\mathcal{A}+\mathcal{C}) & h_{13} \phi \mathcal{A}+\omega \psi \mathcal{C} & h_{13}^{2} \mathcal{A}+\omega^{2} \mathcal{C}
\end{array}\right)
$$

this observation is enough to analyze its asymptotic behavior.

Theorem 2.10. The quasi-convergence class $[g]$ of an arbitrary solv-geometry metric $g$ is exactly a 4-parameter family.

Proof. Without loss of generality, we may assume that with respect to a frame field for which all structure constants vanish except $c_{23}^{1}=-2$ and 
$c_{12}^{3}=2, g$ is represented by a diagonal matrix corresponding to the initial data $\left(A_{0}, B_{0}, C_{0}\right)$. Since

$$
\begin{aligned}
|h-g|_{g}^{2}= & \frac{\left(\omega^{2} \mathcal{A}+h_{13}^{2} \mathcal{C}-A\right)^{2}}{A^{2}}+\frac{\left(\phi^{2} \mathcal{A}+\mathcal{B}+\psi^{2} \mathcal{C}-B\right)^{2}}{B^{2}} \\
& +\frac{\left(h_{13}^{2} \mathcal{A}+\omega^{2} \mathcal{C}-C\right)^{2}}{C^{2}}+2 \frac{\left(\omega \phi \mathcal{A}+h_{13} \psi \mathcal{C}\right)^{2}}{A B} \\
& +2 \frac{\left(\omega h_{13}(\mathcal{A}+\mathcal{C})\right)^{2}}{A C}+2 \frac{\left(h_{13} \phi \mathcal{A}+\omega \psi \mathcal{C}\right)^{2}}{B C}
\end{aligned}
$$

and $A, C \rightarrow \sqrt{A_{0} C_{0}}$, it is clear that $h \in[g]$ only if $h_{13}=0$.

If $h_{13}=0$, then $\omega^{2} \mathcal{A}$ and $\omega^{2} \mathcal{C}$ both converge to $\sqrt{h_{11} h_{33}}$ and

$$
\begin{aligned}
|h-g|_{g}^{2}= & \frac{\left(\omega^{2} \mathcal{A}-A\right)^{2}}{A^{2}}+\frac{\left(\left(h_{12} / h_{11}\right)^{2} \omega^{2} \mathcal{A}+\left(h_{23} / h_{33}\right)^{2} \omega^{2} \mathcal{C}+\mathcal{B}-B\right)^{2}}{B^{2}} \\
& +\frac{\left(\omega^{2} \mathcal{C}-C\right)^{2}}{C^{2}}+2 \frac{\left(\left(h_{12} / h_{11}\right) \omega^{2} \mathcal{A}\right)^{2}}{A B}+2 \frac{\left(\left(h_{23} / h_{33}\right) \omega^{2} \mathcal{C}\right)^{2}}{B C} .
\end{aligned}
$$

Since $B \rightarrow \infty$ and by de l'Hôpital,

$$
\lim _{t \rightarrow \infty} \frac{\mathcal{B}}{B}=\lim _{t \rightarrow \infty} \frac{\left(\omega^{2} \mathcal{A}+\omega^{2} \mathcal{C}\right)^{2} A C}{(A+C)^{2} \omega^{2} \mathcal{A} \omega^{2} \mathcal{C}}=1
$$

it is easy to see that $h \in[g]$ if only if $h_{13}=0$ and $h_{11} h_{33}=A_{0} C_{0}$.

\subsection{The geometry of $\widetilde{\mathrm{SL}(2, \mathbb{R})}$.}

2.5.1. The class $[g]_{\mathcal{F}}$ for an $\widehat{\mathrm{SL}(2, \mathbb{R})}$-geometry metric $g$. Consider $(\mathcal{G}, g)$, where $\mathcal{G}=\mathrm{SL}(2, \mathbb{R}),\left\{F_{i}\right\}$ is a Milnor frame with dual forms $\left\{\omega^{i}\right\}$, and the metric is

$$
g=A \omega^{1} \otimes \omega^{1}+B \omega^{2} \otimes \omega^{2}+C \omega^{3} \otimes \omega^{3} .
$$

We may take $\lambda^{1}=-1$ and $\lambda^{2}=\lambda^{3}=1$. Then the sectional curvatures are

$$
\begin{aligned}
& K\left(F_{2} \wedge F_{3}\right)=\frac{(B-C)^{2}-A(3 A+2 B+2 C)}{A B C} \\
& K\left(F_{3} \wedge F_{1}\right)=\frac{[A-(B-C)]^{2}-4 B(B-C)}{A B C} \\
& K\left(F_{1} \wedge F_{2}\right)=\frac{[A+(B-C)]^{2}+4 C(B-C)}{A B C} .
\end{aligned}
$$


If we write $A_{0} \doteqdot A(0), B_{0} \doteqdot B(0)$, and $C_{0} \doteqdot C(0)$, the Ricci flow is equivalent to the system

$$
\begin{aligned}
& \frac{d}{d t} A=4 \frac{(B-C)^{2}-A^{2}}{B C} \\
& \frac{d}{d t} B=4 \frac{(A+C)^{2}-B^{2}}{A C} \\
& \frac{d}{d t} C=4 \frac{(A+B)^{2}-C^{2}}{A B} .
\end{aligned}
$$

If $B_{0}=C_{0}$, the flow preserves the equality $B=C$ and conserves the quantity

$$
\Phi \doteqdot \frac{A^{2} B}{A+B}
$$

In this case, $A^{\prime}=-4 A^{2} / B^{2}<0$ and $B^{\prime}=8+4 A / B>8$, whence it follows easily that $A^{\prime} \nearrow 0$ and $B^{\prime} \equiv C^{\prime} \searrow 8$ as $t \rightarrow \infty$. So $B \equiv C \sim 8 t$ and

$$
\lim _{t \rightarrow \infty} A(t)=\lim _{t \rightarrow \infty} \frac{\Phi+\sqrt{\Phi^{2}+4 \Phi B^{2}}}{2 B}=\sqrt{\Phi}>0 .
$$

It would be very useful if there were a conserved quantity in the general case. Our inability to find any such quantity forces us to use alternate methods in analyzing the quasi-convergence behavior of $\widetilde{\mathrm{SL}(2, \mathbb{R})}$ metrics. Define $\mathcal{O}_{+} \doteqdot\left\{(a, b, c) \in \mathbb{R}^{3}: a>0, b>0, c>0\right\}$ and $\mathbb{R}_{+} \doteqdot\{a \in \mathbb{R}: a>0\}$. Consider a typical cube $\mathcal{K} \subset \mathcal{O}_{+}$defined by

$$
\begin{aligned}
\mathcal{K}\left(a_{*}, a^{*}, b_{*}, b^{*}, c_{*}, c^{*}\right) & \\
& \doteqdot\left\{(a, b, c) \in \mathcal{O}_{+}: a_{*} \leq a \leq a^{*}, b_{*} \leq b \leq b^{*}, c_{*} \leq c \leq c^{*}\right\}
\end{aligned}
$$

where $a^{*}-a_{*}, b^{*}-b_{*}$, and $c^{*}-c_{*}$ are presumed small.

Lemma 2.11. For any $\mathcal{K} \subset \mathcal{O}_{+}$and any initial data $\left(A_{0}, B_{0}, C_{0}\right) \in \operatorname{int} \mathcal{K}$, there are $t_{*} \geq 0$ and constants $k, K>0$ depending only on $\mathcal{K}$ such that for all $t \geq t_{*}$,

- $A$ decreases monotonically to $A_{\infty}=A_{\infty}\left(A_{0}, B_{0}, C_{0}\right)>0$;

- $B$ and $C$ grow asymptotically like $8 t$; and

- $|B-C| \leq K e^{-k t}$. 
Proof. In considering the system (49a)-(49c), we say that a solution exists as long as $0<A, B, C<\infty$. Notice that $\delta \doteqdot A(B-C)$ satisfies $\delta^{\prime}=-16 \delta / A$. Since the solution $\delta \equiv 0$ is unique, we may assume that $B_{0} \geq C_{0}$. (The arguments for the case $B_{0}<C_{0}$ are entirely analogous.) The proof comprises five claims.

Claim 2.12. A solution exists as long as $0<A<\infty$; any such solution has the property that

$$
c_{*}+8 t \leq C \leq B \leq b^{*}+\left(8+4 \frac{a^{*}}{c_{*}}\right) t .
$$

Noting that $B \geq C$ for as long as a solution exists, we have

$$
C^{\prime}=4 \frac{A^{2}+2 A B+B^{2}-C^{2}}{A B} \geq 8
$$

and

$$
\left(\frac{A}{C}\right)^{\prime}=8 \frac{C^{2}-B C-A^{2}-A B}{B C^{2}} \leq 0
$$

which lets us estimate

$$
B^{\prime}=4 \frac{A^{2}+2 A C+C^{2}-B^{2}}{A C} \leq 8+4 \frac{A}{C} \leq 8+4 \frac{A_{0}}{C_{0}} \leq 8+4 \frac{a^{*}}{c_{*}} .
$$

Claim 2.13. A solution exists at least to some $t_{0}=t_{0}(\mathcal{K})>0$ with the property that $A \leq B+C$ for all $t \geq t_{0}$. Hence a solution exists as long as $A>0$.

If $A_{0}>B_{0}+C_{0}$, then for as long as $A>B+C$, we have

$$
A^{\prime}=4 \frac{(B-C)^{2}-A^{2}}{B C} \leq-16
$$

so that $A(t) \leq A_{0}-16 t \leq a^{*}-16 t$. And for the same times, we have

$$
(B+C)^{\prime}=4\left[\frac{(A+C)^{2}-B^{2}}{A C}+\frac{(A+B)^{2}-C^{2}}{A B}\right] \geq \frac{32}{a^{*}}(B+C),
$$

so that $B+C \geq\left(b_{*}+c_{*}\right) e^{32 t / a^{*}}$. Clearly, the inequality $A>B+C$ cannot persist, and a solution exists up to some $t_{0}=t_{0}(\mathcal{K})$ when $A=B+C>0$.

On the other hand, if $A=B+C$ at any $t \geq 0$, then $A^{\prime}=-16$ and $(B+C)^{\prime}=32$ at $t$, so immediately $A<B+C$. 
Claim 2.14. A solution exists at least to some $t_{1}=t_{1}(\mathcal{K})>t_{0}$ with the property that $A \geq B-C$ for all $t \geq t_{1}$ such that $A>0$.

If $A\left(t_{0}\right)<B\left(t_{0}\right)-C\left(t_{0}\right)$, then $A^{\prime}>0$ as long as $A<B-C$, whence $A$ remains positive. But since $B>C$ as long as a solution exists and

$$
(B-C)^{\prime}=\frac{4}{A B C}\left[A^{2}-(B+C)^{2}\right](B-C)<\frac{-16}{A}(B-C)<-16,
$$

the inequality $A<B-C$ cannot last. Hence a solution exists at least up to some $t_{1}=t_{1}(\mathcal{K})>t_{0}$ when $A=B-C>0$.

On the other hand, if $A=B-C$ at any $t \geq 0$, we have $A^{\prime}=0$ and $(B-C)^{\prime}=-16$ at $t$, so immediately $A>B-C$.

Claim 2.15. For all $t \geq t_{1}, A$ is monotone decreasing to

$$
A_{\infty} \geq \frac{2 A\left(t_{1}\right)\left(c_{*}+8 t_{1}\right)}{A\left(t_{1}\right)+2\left(c_{*}+8 t_{1}\right)}>0 .
$$

Hence a solution exists for all time.

For all $t \geq t_{1}$ such that a solution exists, we can estimate

$$
0 \geq A^{\prime}=4 \frac{(B-C)^{2}-A^{2}}{B C} \geq-4 \frac{A^{2}}{B C} \geq-4 \frac{A^{2}}{\left(c_{*}+8 t\right)^{2}} .
$$

Integrating this inequality shows that

$$
0 \geq \frac{1}{A\left(t_{1}\right)}-\frac{1}{A} \geq \frac{4\left(t_{1}-t\right)}{\left(c_{*}+8 t\right)\left(c_{*}+8 t_{1}\right)} .
$$

Because the RHS converges to $-1 / 2\left(c_{*}+8 t_{1}\right)$ as $t \rightarrow \infty, A$ decreases monotonically to some $A_{\infty}$ such that

$$
A_{\infty} \geq \frac{2 A\left(t_{1}\right)\left(c_{*}+8 t_{1}\right)}{A\left(t_{1}\right)+2\left(c_{*}+8 t_{1}\right)}>0 .
$$

Claim 2.16. $B$ and $C$ grow asymptotically like $8 t$, and there are $t_{*}=$ $t_{*}(\mathcal{K}) \geq t_{1}$ and $k=k(\mathcal{K})>0$ such that for $t \geq t_{*}$,

$$
(B-C) \leq\left(B\left(t_{*}\right)-C\left(t_{*}\right)\right) e^{-k t} .
$$


To prove the first statement, recall that $c_{*}+8 t \leq C \leq B$, whence it follows from estimate (51) that for $t \geq t_{1}$,

$$
B^{\prime} \leq 8+4 \frac{A}{C} \leq 8+4 \frac{\sup _{\left(A_{0}, B_{0}, C_{0}\right) \in \mathcal{K} A\left(t_{1}\right)}}{c_{*}+8 t} \rightarrow 8 \text { as } \quad t \rightarrow \infty .
$$

To prove the second, recall that

$$
A\left(t_{1}\right) \leq(B+C)\left(t_{1}\right) \leq 2\left(b^{*}+\left(8+4 \frac{a^{*}}{c_{*}}\right) t_{1}\right)
$$

and $C \geq c_{*}+8 t$. So there is some $t_{*}=t_{*}(\mathcal{K}) \geq t_{1}$ such that $A<C$ at $t_{*}$. Then for all later times, we have

$$
(B-C)^{\prime}=4 \frac{\left(A^{2}-C^{2}\right)-\left(B^{2}+2 B C\right)}{A B C}(B-C)<-\frac{12}{A\left(t_{*}\right)}(B-C) .
$$

So for such times, $(B-C) \leq K e^{-k t}$, where $k, K$ depend only on $\mathcal{K}$. (In fact, it is easy to see that $(B-C)^{\prime} \rightarrow-16(B-C) / A_{\infty}$ as $t \rightarrow \infty$.)

Lemma 2.17. The map $\Omega: \mathcal{O}_{+} \rightarrow \mathbb{R}_{+}$defined by

$$
\Omega:\left(A_{0}, B_{0}, C_{0}\right) \mapsto A_{\infty}
$$

is homogeneous of degree 1.

Proof. If $\lambda>0$ and we denote a solution to the system (49a )-(49c) by

$$
t \mapsto(A(t), B(t), C(t)) \in \mathcal{O}_{+}
$$

then

$$
t \mapsto\left(\lambda A\left(\frac{t}{\lambda}\right), \lambda B\left(\frac{t}{\lambda}\right), \lambda C\left(\frac{t}{\lambda}\right)\right) \in \mathcal{O}_{+}
$$

is also a solution. Hence it suffices to observe that

$$
\Omega\left(A_{0}, B_{0}, C_{0}\right)=\lim _{t \rightarrow \infty} A(t)=A_{\infty}
$$

while

$$
\Omega\left(\lambda A_{0}, \lambda B_{0}, \lambda C_{0}\right)=\lim _{t \rightarrow \infty} \lambda A\left(\frac{t}{\lambda}\right)=\lambda A_{\infty}
$$


Corollary 2.18. The set $\Omega^{-1}(1)$ intersects every ray from the origin in $\mathcal{O}_{+}$ exactly once.

Proof. Let $\rho$ be an arbitrary ray, say from $0 \in \mathbb{R}^{3}$ through $\left(A_{0}, B_{0}, C_{0}\right) \in \mathcal{O}_{+}$. Let $\omega \doteqdot \Omega\left(A_{0}, B_{0}, C_{0}\right)>0$. Then by the lemma,

$$
\Omega\left(\frac{1}{\omega} A_{0}, \frac{1}{\omega} B_{0}, \frac{1}{\omega} C_{0}\right)=1 .
$$

So $\Omega^{-1}(1)$ intersects $\rho$ at least once. On the other hand, if $\Omega(a, b, c)=1$ for some $(a, b, c) \in \mathcal{O}_{+}$on $\rho$, then $\Omega(\lambda a, \lambda b, \lambda c)=\lambda \neq 1$ for any positive $\lambda \neq 1$. So $\Omega^{-1}(1)$ intersects $\rho$ at most once.

Corollary 2.19. The class $[g]_{\mathcal{F}}$ for an $\widetilde{\mathrm{SL}(2, \mathbb{R})}$-geometry metric is a 2 parameter family.

Proof. By Claim 2.16 and de l'Hôpital, $[g]_{\mathcal{F}}$ depends only on $A_{\infty}$.

We will next show that $[g]_{\mathcal{F}}$ is a smooth 2-parameter family. We shall use a theorem of N. Levinson on asymptotically diagonal linear systems. An examination of the proof given in [2] allows us to state the result in a form adapted to our purposes:

Theorem 2.20 (The Levinson theorem). Let $\Lambda$ and $\Gamma$ be continuous real-valued matrix functions on a half line $t_{0} \leq t<\infty$. Suppose $\Lambda=$ $\operatorname{diag}\left(\lambda_{1}, \ldots, \lambda_{n}\right)$ is diagonal, and $\Gamma$ obeys the integrability condition that $|\Gamma(t)| \leq \gamma(t)$ for some $\gamma \in L^{1}\left(\left[t_{0}, \infty\right)\right)$. We say an eigenvalue $\lambda_{j}$ satisfies the dichotomy condition if there exist $\beta_{*} \leq \beta^{*}:\left[t_{0}, \infty\right) \rightarrow \mathbb{R}$ such that $\beta_{*} \leq 0$ and $\int_{t_{0}}^{\infty} \beta^{*}(s) d s=-\infty$ and constants $K_{1}$ and $K_{2}$ such that each $\lambda_{i}(i=1, \ldots, n)$ has one of the following mutually exclusive properties:

(D1) $\int_{t_{1}}^{t_{2}}\left(\lambda_{i}(t)-\lambda_{j}(t)\right) d t \leq K_{1}$ for all intervals $\left[t_{1}, t_{2}\right] \subset\left[t_{0}, \infty\right)$ and $\beta_{*}(t) \leq \lambda_{i}(t)-\lambda_{j}(t) \leq \beta^{*}(t)$ for all $t \in\left[t_{0}, \infty\right) ;$

(D2) $\int_{t_{1}}^{t_{2}}\left(\lambda_{i}(t)-\lambda_{j}(t)\right) d t \geq K_{2}$ for all intervals $\left[t_{1}, t_{2}\right] \subset\left[t_{0}, \infty\right)$.

Then for each $\lambda_{j}$ satisfying the dichotomy condition, there is $t_{1} \in\left[t_{0}, \infty\right)$ depending only on $\gamma, K_{1}, K_{2}$, and $n$ such that system

$$
V^{\prime}(t)=(\Lambda(t)+\Gamma(t)) V(t)
$$


has a solution $V_{j}$ defined for $t_{1} \leq t<\infty$ of the form

$$
V_{j}(t)=\left(e_{j}+\Psi(t)\right) \exp \int_{t_{1}}^{t} \lambda_{j}(s) d s .
$$

Here $e_{j} \in \mathbb{R}^{n}$ is the vector with components $\delta_{i j}$, and $\Psi(t) \in \mathbb{R}^{n}$ has the property that for every $\varepsilon>0$, there is $T_{\varepsilon} \geq t_{1}$ depending only on $\varepsilon, \beta_{*}, \beta^{*}$, $\gamma, K_{1}, K_{2}$, and $n$ such that $\sup _{t \geq T_{\varepsilon}}|\Psi(t)| \leq \varepsilon$.

Our main result on $\widetilde{\mathrm{SL}(2, \mathbb{R})}$-metrics is:

Theorem 2.21. $\Omega^{-1}(a)$ is a 2-dimensional submanifold of $\mathcal{O}_{+}$for every $a>0$.

Proof. The argument consists of three claims:

Claim 2.22. $\Omega$ is continuous.

For each $t \geq 0$, define a map $\Omega_{t}: \mathcal{O}_{+} \rightarrow \mathbb{R}_{+}$by $\Omega_{t}:\left(A_{0}, B_{0}, C_{0}\right) \mapsto A(t)$. Then for any cube $\mathcal{K} \subset \mathcal{O}_{+}$and any solution $(A, B, C)$ with initial data in $\mathcal{K}$, it follows from Lemma 2.11 that there is $k>0$ depending only on $\mathcal{K}$ such that for all $t_{2}>t_{1} \geq t_{*}(\mathcal{K})$

$$
\begin{aligned}
\left|\Omega_{t_{2}}-\Omega_{t_{1}}\right| & =\left|\int_{t_{1}}^{t_{2}} A^{\prime}(t) d t\right| \\
& \leq \int_{t_{1}}^{t_{2}} 4 \frac{\left|(B-C)^{2}-A^{2}\right|}{B C} d t \leq \int_{t_{1}}^{t_{2}} \frac{k}{t^{2}} d t=\frac{k}{t_{1}}-\frac{k}{t_{2}} .
\end{aligned}
$$

Hence $\Omega_{t} \rightarrow \Omega$ locally uniformly in $\mathcal{O}_{+}$, which proves the claim.

Claim 2.23. $\Omega$ is continuously differentiable.

Consider a solution $(A, B, C)$ with initial data $\left(A_{0}, B_{0}, C_{0}\right) \in \operatorname{int} \mathcal{K}$ for some $\mathcal{K} \subset \mathcal{O}_{+}$. We shall prove that $\partial A / \partial A_{0}, \partial A / \partial B_{0}$, and $\partial A / \partial C_{0}$ converge to finite limits as $t \rightarrow \infty$ uniformly with respect to $\mathcal{K}$. Let $E \doteqdot B+C$ and $F \doteqdot B-C$ and define

$$
P(t) \doteqdot\left(\begin{array}{lll}
\partial A / \partial A_{0} & \partial A / \partial B_{0} & \partial A / \partial C_{0} \\
\partial E / \partial A_{0} & \partial E / \partial B_{0} & \partial E / \partial C_{0} \\
\partial F / \partial A_{0} & \partial F / \partial B_{0} & \partial F / \partial C_{0}
\end{array}\right) .
$$


Then $P$ satisfies the linear system

$$
\left\{\begin{array}{l}
P^{\prime}=Q P \\
P(0)=\left(\begin{array}{ccc}
1 & 0 & 0 \\
0 & 1 & 1 \\
0 & 1 & -1
\end{array}\right),
\end{array}\right.
$$

where it is a straightforward task to compute

$$
\begin{aligned}
Q_{11} & =-\frac{8 A}{B C} \\
Q_{12} & =\frac{2}{B^{2} C^{2}}(B+C)\left(A^{2}-(B-C)^{2}\right) \\
Q_{13} & =-\frac{2}{B^{2} C^{2}}(B-C)\left(A^{2}-(B+C)^{2}\right)
\end{aligned}
$$

and

$$
\begin{aligned}
& Q_{21}=\frac{4}{A^{2} B C}(B+C)\left(A^{2}+(B-C)^{2}\right) \\
& Q_{22}=-\frac{2}{A B^{2} C^{2}}\left(B^{2}+C^{2}\right)\left(A^{2}-(B-C)^{2}\right) \\
& Q_{23}=\frac{2}{A B^{2} C^{2}}\left(B^{2}-C^{2}\right)\left(A^{2}-(B+C)^{2}\right)
\end{aligned}
$$

and

$$
\begin{aligned}
& Q_{31}=\frac{4}{A^{2} B C}(B-C)\left(A^{2}+(B+C)^{2}\right) \\
& Q_{32}=-\frac{2}{A B^{2} C^{2}}\left(B^{2}-C^{2}\right)\left(A^{2}-(B-C)^{2}\right) \\
& Q_{33}=\frac{2}{A B^{2} C^{2}}\left(B^{2}+C^{2}\right)\left(A^{2}-(B+C)^{2}\right) .
\end{aligned}
$$

Let $t_{*}=t_{*}(\mathcal{K})$ be the time given by Lemma 2.11. Since $A\left(t_{*}\right)$ is a continuous function of $\left(A_{0}, B_{0}, C_{0}\right) \in \mathcal{K}$, there are by Claim 2.15 constants $k_{1}, k_{2}>0$ depending only on $\mathcal{K}$ such that

$$
k_{1} \leq A \leq k_{2}
$$

for all $t \geq t_{*}$. By Claim 2.12, there are $k_{3}, \ldots, k_{6}>0$ depending only on $\mathcal{K}$ such that

$$
k_{3}+k_{4} t \leq B \leq k_{5}+k_{6} t \quad \text { and } \quad k_{3}+k_{4} t \leq C \leq k_{5}+k_{6} t
$$


for all $t \geq 0$. And by Claim 2.16, there are $k_{7}, k_{8}>0$ depending only on $\mathcal{K}$ such that

$$
|B-C| \leq k_{7} e^{-k_{8} t}
$$

for all $t \geq t_{*}$. Hence there are $c_{1}, \ldots, c_{4}>0$ depending only on $\mathcal{K}$ such that the off-diagonal components of $Q$ obey the following estimates for all $t \geq t_{*}$ :

$$
\begin{aligned}
\left|Q_{12}\right| & \leq 2 B^{-2} C^{-2}(B+C)\left(A^{2}+(B-C)^{2}\right) \\
& \leq 4\left(k_{3}+k_{4} t\right)^{-4}\left(k_{5}+k_{6} t\right)\left(k_{2}^{2}+k_{7}^{2} e^{-2 k_{8} t}\right) \leq \frac{c_{1}}{c_{2}+t^{3}} \\
\left|Q_{21}\right| & \leq 4 A^{-2} B^{-1} C^{-1}(B+C)\left(A^{2}+(B-C)^{2}\right) \\
& \leq 8 k_{1}^{-2}\left(k_{3}+k_{4} t\right)^{-2}\left(k_{5}+k_{6} t\right)\left(k_{2}^{2}+k_{7}^{2} e^{-2 k_{8} t}\right) \leq \frac{c_{1}}{c_{2}+t} \\
\left|Q_{13}\right| & \leq 2 B^{-2} C^{-2}|B-C|\left(A^{2}+(B+C)^{2}\right) \leq c_{3} e^{-c_{4} t} \\
\left|Q_{31}\right| & \leq 4 A^{-2} B^{-1} C^{-1}|B-C|\left(A^{2}+(B+C)^{2}\right) \leq c_{3} e^{-c_{4} t} \\
\left|Q_{23}\right| & \leq 2 A^{-1} B^{-2} C^{-2}|B-C|(B+C)\left(A^{2}+(B+C)^{2}\right) \leq c_{3} e^{-c_{4} t} \\
\left|Q_{32}\right| & \leq 2 A^{-1} B^{-2} C^{-2}|B-C|(B+C)\left(A^{2}+(B-C)^{2}\right) \leq c_{3} e^{-c_{4} t}
\end{aligned}
$$

We estimate the diagonal components of $Q$ for $t \geq t_{*}$ as follows:

$$
\begin{aligned}
-\frac{8 k_{2}}{\left(k_{3}+k_{4} t\right)^{2}} & \leq Q_{11} \leq-\frac{8 k_{1}}{\left(k_{5}+k_{6} t\right)^{2}} \\
-\frac{4 k_{2}\left(k_{5}+k_{6} t\right)^{2}}{\left(k_{3}+k_{4} t\right)^{4}} & \leq Q_{22} \leq \frac{4\left(k_{5}+k_{6} t\right)^{2} k_{7}^{2} e^{-2 k_{8} t}}{k_{1}\left(k_{3}+k_{4} t\right)^{4}} \\
-\frac{16\left(k_{5}+k_{6} t\right)^{4}}{k_{1}\left(k_{3}+k_{4} t\right)^{4}} & \leq Q_{33} \leq \frac{4 k_{2}\left(k_{5}+k_{6} t\right)^{2}}{\left(k_{3}+k_{4} t\right)^{4}}-\frac{8}{k_{2}} .
\end{aligned}
$$

(The last inequality follows from the fact that $\left(B^{2}+C^{2}\right)(B+C)^{2} \geq$ $4 B^{2} C^{2}$.)

Now put $T \doteqdot \operatorname{diag}(1,1 / t, 1)$ and $W \doteqdot T P$. Consider the system

$$
W^{\prime}=\left(T^{\prime} T^{-1}+T Q T^{-1}\right) W=(\Lambda+\Gamma) W
$$

defined for $t \geq t_{0}$, where $t_{0} \geq t_{*}$ is to be determined,

$$
\Lambda(t)=\operatorname{diag}\left(\lambda_{1}(t), \lambda_{2}(t), \lambda_{3}(t)\right) \doteqdot\left(\begin{array}{ccc}
Q_{11} & & \\
& Q_{22}-1 / t & \\
& & Q_{33}
\end{array}\right)
$$


and

$$
\Gamma(t) \doteqdot\left(\begin{array}{ccc}
0 & Q_{12} t & Q_{13} \\
Q_{21} / t & 0 & Q_{23} / t \\
Q_{31} & Q_{32} t & 0
\end{array}\right)
$$

Notice that $\lambda_{1}$ behaves like a constant multiple of $-1 / t^{2}, \lambda_{2}$ behaves like $-1 / t$, and $\lambda_{3}$ behaves like a negative constant. Choose $t_{0} \geq k_{2} / 4$ and make the following four observations:

- $\Gamma$ obeys the integrability condition of Theorem 2.20 uniformly with respect to solutions whose initial values lie in $\mathcal{K}$. Indeed, it follows from the estimates obtained above for $\left|Q_{i j}\right|(i \neq j)$ that there exists a positive continuous function $\gamma \in L^{1}\left(\left[t_{*}, \infty\right)\right)$ depending only on $\mathcal{K}$ and $n$ such that $|\Gamma(t)| \leq \gamma(t)$ for all $t \geq t_{0} \geq t_{*}$.

- The eigenvalue $\lambda_{1}$ of $\Lambda$ obeys the dichotomy condition of Theorem 2.20 uniformly. Indeed, we deduce from the estimates for $Q_{i i}$ that there are constants $c_{5}, c_{6}>0$ depending only on $\mathcal{K}$ such that

$$
\begin{aligned}
& \lambda_{2}-\lambda_{1} \geq-\frac{4 k_{2}\left(k_{5}+k_{6} t\right)^{2}}{\left(k_{3}+k_{4} t\right)^{4}}-\frac{1}{t} \\
& \lambda_{2}-\lambda_{1} \leq \frac{4\left(k_{5}+k_{6} t\right)^{2} k_{7}^{2} e^{-2 k_{8} t}}{k_{1}\left(k_{3}+k_{4} t\right)^{4}}+\frac{8 k_{2}}{\left(k_{3}+k_{4} t\right)^{2}}-\frac{1}{t} \leq \frac{c_{5}}{c_{6}+t^{2}}-\frac{1}{t}
\end{aligned}
$$

and

$$
\begin{aligned}
& \lambda_{3}-\lambda_{1} \geq-\frac{16\left(k_{5}+k_{6} t\right)^{4}}{k_{1}\left(k_{3}+k_{4} t\right)^{4}} \\
& \lambda_{3}-\lambda_{1} \leq \frac{4 k_{2}\left(k_{5}+k_{6} t\right)^{2}}{\left(k_{3}+k_{4} t\right)^{4}}-\frac{8}{k_{2}}+\frac{8 k_{2}}{\left(k_{3}+k_{4} t\right)^{2}} \leq \frac{c_{5}}{c_{6}+t^{2}}-\frac{8}{k_{2}} .
\end{aligned}
$$

Hence both $\lambda_{2}$ and $\lambda_{3}$ satisfy property (D1) if we set

$$
\begin{aligned}
K_{1} & =K \doteqdot \int_{t_{0}}^{\infty} \frac{c_{5}}{c_{6}+t^{2}} d t<\infty \\
\beta_{*}(t) & =-\frac{4 k_{2}\left(k_{5}+k_{6} t\right)^{2}}{\left(k_{3}+k_{4} t\right)^{4}}-\frac{1}{t}-\frac{16\left(k_{5}+k_{6} t\right)^{4}}{k_{1}\left(k_{3}+k_{4} t\right)^{4}} \\
\beta^{*}(t) & =\frac{c_{5}}{c_{6}+t^{2}}-\frac{1}{t}
\end{aligned}
$$


- The eigenvalue $\lambda_{2}$ obeys the dichotomy condition uniformly. Indeed, since

$$
\lambda_{1}-\lambda_{2} \geq-\frac{c_{5}}{c_{6}+t^{2}}+\frac{1}{t}
$$

$\lambda_{1}$ satisfies property (D2) with $K_{2}=-K$. And because there is $c_{7}>0$ depending only on $\mathcal{K}$ such that

$$
\begin{aligned}
& \lambda_{3}-\lambda_{2} \geq-\frac{16\left(k_{5}+k_{6} t\right)^{4}}{k_{1}\left(k_{3}+k_{4} t\right)^{4}}-\frac{4\left(k_{5}+k_{6} t\right)^{2} k_{7}^{2} e^{-2 k_{8} t}}{k_{1}\left(k_{3}+k_{4} t\right)^{4}}+\frac{1}{t} \geq-c_{7}+\frac{1}{t} \\
& \lambda_{3}-\lambda_{2} \leq \frac{8 k_{2}\left(k_{5}+k_{6} t\right)^{2}}{\left(k_{3}+k_{4} t\right)^{4}}-\frac{8}{k_{2}}+\frac{1}{t} \leq \frac{2 c_{5}}{c_{6}+t^{2}}-\frac{4}{k_{2}},
\end{aligned}
$$

$\lambda_{3}$ satisfies property (D1) with

$$
K_{1}=2 K, \quad \beta_{*}(t)=-c_{7}, \quad \beta^{*}(t)=\frac{2 c_{5}}{c_{6}+t^{2}}-\frac{4}{k_{2}} .
$$

- The eigenvalue $\lambda_{3}$ obeys the dichotomy condition uniformly. Indeed, since

$$
\lambda_{1}-\lambda_{3} \geq-\frac{c_{5}}{c_{6}+t^{2}}+\frac{8}{k_{2}} \quad \text { and } \quad \lambda_{2}-\lambda_{3} \geq-\frac{2 c_{5}}{c_{6}+t^{2}}+\frac{4}{k_{2}},
$$

both $\lambda_{1}$ and $\lambda_{2}$ satisfy property (D2) with $K_{2}=-2 K$.

Recalling that $\lambda_{1}, \lambda_{2}$, and $\lambda_{3}$ are bounded uniformly with respect to $\mathcal{K}$, we apply Theorem 2.20 to system (54), obtaining a time $t_{1} \geq t_{0}$ depending only on $\mathcal{K}$ and vector solutions

$$
\begin{aligned}
& V_{1}(t)=\left(\left(\begin{array}{l}
1 \\
0 \\
0
\end{array}\right)+o(1)\right) \exp \int_{t_{1}}^{t} \lambda_{1}(s) d s \rightarrow\left(\begin{array}{c}
\exp \int_{t_{1}}^{\infty} \lambda_{1}(s) d s \\
0 \\
0
\end{array}\right) \\
& V_{2}(t)=\left(\left(\begin{array}{l}
0 \\
1 \\
0
\end{array}\right)+o(1)\right) \exp \int_{t_{1}}^{t} \lambda_{2}(s) d s \rightarrow\left(\begin{array}{l}
0 \\
0 \\
0
\end{array}\right) \\
& V_{3}(t)=\left(\left(\begin{array}{l}
0 \\
0 \\
1
\end{array}\right)+o(1)\right) \exp \int_{t_{1}}^{t} \lambda_{3}(s) d s \rightarrow\left(\begin{array}{l}
0 \\
0 \\
0
\end{array}\right)
\end{aligned}
$$


defined for $t \geq t_{1}$. Note that $e^{\int_{t_{1}}^{\infty} \lambda_{1}(s) d s}$ can be estimated by constants depending only on $\mathcal{K}$ and that everything in sight converges uniformly with respect to $\mathcal{K}$. Note also that the set $\left\{V_{1}(t), V_{2}(t), V_{3}(t)\right\}$ is linearly independent. Indeed, suppose $\alpha^{k} V_{k}(t) \equiv 0$ for some constants $\alpha^{1}, \alpha^{2}, \alpha^{3}$. Then as $t \rightarrow \infty$,

$$
\alpha^{1}=o(1)-\alpha^{2}(o(1)) e^{\int_{t_{1}}^{t}\left(\lambda_{2}-\lambda_{1}\right) d s}-\alpha^{3}(o(1)) e^{\int_{t_{1}}^{t}\left(\lambda_{3}-\lambda_{1}\right) d s} .
$$

By the estimates above for $\lambda_{2}-\lambda_{1}$ and $\lambda_{3}-\lambda_{1}$, this is possible only if $\alpha^{1}=0$, hence only if

$$
\alpha^{2}=o(1)-\alpha^{3}(o(1)) e^{\int_{t_{1}}^{t}\left(\lambda_{3}-\lambda_{2}\right) d s} .
$$

By the estimate for $\lambda_{3}-\lambda_{2}$, this is possible only if $\alpha^{2}=0$, hence only if $\alpha^{1}=\alpha^{2}=\alpha^{3}=0$. Therefore there are constants $\alpha_{i j}^{k}$ depending only on $W\left(t_{1}\right)$ such that $W_{i j}(t)=\alpha_{i j}^{k} V_{k}(t)$ for all $t \geq t_{1}$. Because all $\alpha_{i j}^{k}$ can be estimated uniformly with respect to $\mathcal{K}$, we conclude that $\partial A / \partial A_{0}, \partial A / \partial B_{0}$, and $\partial A / \partial C_{0}$ converge to finite limits locally uniformly with respect to initial conditions.

Claim 2.24. $\nabla \Omega$ is never degenerate.

The fact that $\Omega(\lambda a, \lambda b, \lambda c)=\lambda \Omega(a, b, c)$ for all $(a, b, c) \in \mathcal{O}_{+}$and $\lambda>0$ proves $\left.\nabla \Omega\right|_{(a, b, c)}(a, b, c)=\Omega(a, b, c)>0$.

As we indicated in the introduction, the computations needed to determine the class $[g]$ are formidable. Moreover, we may not be able to apply them without a more explicit understanding of the map $\Omega$. Hence we shall not pursue our analysis of $\widehat{\mathrm{SL}(2, \mathbb{R})}$ metrics any further here.

\section{The geometries not modeled by a Lie group .}

Denote the upper half-space in $\mathbb{R}^{n}$ by $\mathcal{H}^{n}=\left\{(x, y) \in \mathbb{R}^{n}: x \in \mathbb{R}^{n-1}, y>0\right\}$, and let $\operatorname{Conf}\left(\mathcal{H}^{n}\right)$ denote the group of all conformal diffeomorphisms of $\mathcal{H}^{n}$. Let $\mathcal{I}^{n}$ be the subgroup of $\operatorname{Conf}\left(\mathcal{H}^{n}\right)$ generated by $\left\{\tau_{u}: u \in \mathbb{R}^{n-1}\right\}$ and $\left\{\rho_{v}: v>0\right\}$, where $\tau_{u}$ is the translation

$$
\tau_{u}:(x, y) \mapsto(x+u, y)
$$

and $\rho_{v}$ is the radial dilation

$$
\rho_{v}:(x, y) \mapsto(v x, v y) .
$$


For $u_{1}, u_{2} \in \mathbb{R}^{n-1}$ and $v_{1}, v_{2}>0$, we readily verify the relations

$$
\tau_{u_{1}} \tau_{u_{2}}=\tau_{u_{1}+u_{2}}, \quad \rho_{v_{1}} \rho_{v_{2}}=\rho_{v_{1} v_{2}}, \quad \rho_{v} \tau_{u}=\tau_{v u} \rho_{v}, \quad\left(\tau_{u} \rho_{v}\right)^{-1}=\tau_{-u / v} \rho_{1 / v},
$$

whence it follows that

$$
\mathcal{I}^{n}=\left\{\tau_{u} \rho_{v}:(u, v) \in \mathcal{H}^{n}\right\}
$$

It is clear that $\mathcal{I}^{n}$ is a minimal transitive subgroup of $\operatorname{Conf}\left(\mathcal{H}^{n}\right)$, because the isotropy subgroup

$$
\mathcal{I}_{(x, y)}^{n} \doteqdot\left\{\tau_{u} \rho_{v} \in \mathcal{I}^{n}:\left(\tau_{u} \rho_{v}\right)(x, y)=(x, y)\right\}
$$

contains only the identity $\tau_{0} \rho_{1}$ for all $(x, y) \in \mathcal{H}^{n}$. (The authors extend special thanks to Fredric Ancel for suggesting the group $\mathcal{I}^{n}$.)

Now let $p$ be an arbitrary scalar product on $\mathbb{R}^{n}$ and define

$$
g(V, W)(x, y) \doteqdot \frac{p(V, W)}{y^{2}}, \quad(x, y) \in \mathcal{H}^{n}, \quad V, W \in T_{(x, y)} \mathcal{H}^{n}
$$

Note that $g$ agrees with the usual hyperbolic metric when $p$ is the standard inner product, and has the property that each $\left(\tau_{u} \rho_{v}\right)$ is an isometry:

$$
\begin{aligned}
\left(\left(\tau_{u} \rho_{v}\right)^{*} g\right)(V, W)(x, y) & =g\left(D\left(\tau_{u} \rho_{v}\right) V, D\left(\tau_{u} \rho_{v}\right) W\right)\left(\left(\tau_{u} \rho_{v}\right)(x, y)\right) \\
& =v^{2} g(V, W)(v x+u, v y) \\
& =\frac{p(V, W)}{y^{2}}=g(V, W)(x, y) .
\end{aligned}
$$

If $p_{\beta}=\left(p_{i j}\right), p_{\beta}^{-1}=\left(p^{i j}\right)$ in a basis $\beta=\left(\partial / \partial z^{1}, \ldots, \partial / \partial z^{n}\right)$ for $T_{(x, y)} \mathcal{H}^{n}$ induced by any local coordinate system $z^{1}, \ldots, z^{n}$ with $\partial y / \partial z^{i}=\delta_{\text {in }}$, we can write the Riemannian tensor of $g$ as

$$
R_{i j k}^{\ell}=-\frac{p^{n n}}{y^{2}}\left(\delta_{i}^{\ell} p_{j k}-\delta_{j}^{\ell} p_{i k}\right)
$$

and its Ricci tensor as

$$
R_{j k}=-(n-1) p^{n n} g_{j k}
$$

Notice that finding a minimal transitive subgroup of Conf $\left(\mathcal{H}^{n}\right)$ has not yet given us anything essentially new, because $g$ has constant sectional curvature $K=-p^{n n}<0$. (But as we noted in $\S 1$, quasi-convergence asks that two metrics resemble one another without requiring one to be modified by diffeomorphisms.) 


\subsection{The geometry of $\mathbb{H}^{3}$.}

Consider $\mathcal{H}^{3}$ with the homogeneous metric $g$ defined above. We shall regard the Ricci flow $\frac{\partial}{\partial t} g=-2 \mathrm{Rc}$ as an evolution equation for $p$. We may suppose without loss of generality that $p_{\beta}$ is diagonal to start, say $p_{\beta}(0)=\left(\begin{array}{ccc}A_{0} & & \\ & B_{0} & \\ & & C_{0}\end{array}\right)$. Then $p_{\beta}$ remains diagonal and evolves according to the system

$$
\frac{d}{d t} A=4 \frac{A}{C}, \quad \frac{d}{d t} B=4 \frac{B}{C}, \quad \frac{d}{d t} C=4
$$

with solution

$$
A=A_{0}+4 \frac{A_{0}}{C_{0}} t, \quad B=B_{0}+4 \frac{B_{0}}{C_{0}} t, \quad C=C_{0}+4 t .
$$

Notice the conserved quantities

$$
\frac{A}{B}=\Phi \doteqdot \frac{A_{0}}{B_{0}} \quad \text { and } \quad \frac{A B}{C^{2}}=\Psi \doteqdot \frac{A_{0} B_{0}}{C_{0}^{2}}
$$

Now suppose $q$ is another scalar product and $h$ is the homogeneous metric on $\mathcal{H}^{3}$ given by

$$
h(V, W)(x, y) \doteqdot \frac{q(V, W)}{y^{2}}
$$

If $q_{\beta}=\left(q_{i j}\right)$, we set

$$
A \doteqdot\left(\begin{array}{ccc}
1 & -\frac{q_{12}}{q_{11}} & \frac{q_{12} q_{23}-q_{13} q_{22}}{q_{11} q_{22}-q_{12}^{2}} \\
1 & \frac{q_{12} q_{13}-q_{11} q_{23}}{q_{11} q_{22}-q_{12}^{2}} \\
& 1
\end{array}\right)
$$

and make the linear change of coordinates $\left(\begin{array}{lll}z^{1} & z^{2} & z^{3}\end{array}\right)=$ $\left(\begin{array}{lll}x^{1} & x^{2} & y\end{array}\right) A$ (inducing the change of basis $\alpha=\beta A$ in the tangent space) to obtain

$$
q_{\alpha}=\left(\begin{array}{ccc}
Q_{1} & & \\
& Q_{2} / Q_{1} & \\
& & Q_{3} / Q_{2}
\end{array}\right) \doteqdot\left(\begin{array}{ccc}
\mathcal{A}_{0} & & \\
& \mathcal{B}_{0} & \\
& & \mathcal{C}_{0}
\end{array}\right)
$$


where $Q_{n}>0$ is the determinant of the upper left $n \times n$ submatrix of $\left(q_{i j}\right)$. (Compare $\S 2.2 .2$, and notice that $\partial y / \partial z^{i}=\delta_{i n}$ and $\left(q_{\beta}\right)^{n n}=\left(q_{a}\right)^{n n}=$ $Q_{2} / Q_{3}$.) Then the evolution of $h$ by the Ricci flow corresponds to

$$
\left(\begin{array}{ccc}
\mathcal{A} & & \\
& \mathcal{B} & \\
& & \mathcal{C}
\end{array}\right)=\left(\begin{array}{lll}
Q_{1}+4 \frac{Q_{1} Q_{2}}{Q_{3}} t & & \\
& \frac{Q_{2}}{Q_{1}}+4 \frac{Q_{2}^{2}}{Q_{1} Q_{3}} t & \\
& & \frac{Q_{3}}{Q_{2}}+4 t
\end{array}\right) .
$$

Returning to the basis $\beta$, we compute $q_{\beta}=\left(A^{-1}\right)^{\operatorname{tr}} q_{\alpha} A^{-1}$ to be

$$
\left(\begin{array}{ccc}
\mathcal{A} & \frac{q_{12}}{Q_{1}} \mathcal{A} & \frac{q_{13}}{Q_{1}} \mathcal{A} \\
\frac{q_{12}}{Q_{1}} \mathcal{A} & \frac{q_{12}^{2}}{Q_{1}^{2}} \mathcal{A}+\mathcal{B} & \frac{q_{12} q_{13}}{Q_{1}^{2}} \mathcal{A}+\frac{q_{11} q_{23}-q_{12} q_{13}}{Q_{2}} \mathcal{B} \\
\frac{q_{13}}{Q_{1}} \mathcal{A} & \frac{q_{12} q_{13}}{Q_{1}^{2}} \mathcal{A}+\frac{q_{11} q_{23}-q_{12} q_{13}}{Q_{2}} \mathcal{B} & \frac{q_{13}^{2}}{Q_{1}^{2}} \mathcal{A}+\frac{\left(q_{11} q_{23}-q_{12} q_{13}\right)^{2}}{Q_{2}^{2}} \mathcal{B}+\mathcal{C}
\end{array}\right)
$$

Theorem 3.1. The quasi-convergence class $[g]$ of an arbitrary metric $g$ having the geometry of $\mathbb{H}^{3}$ is exactly a 1-parameter family.

Proof. In the notation adopted above, we have $h \in[g]$ if and only iff every term in the sum

$$
\begin{aligned}
|h-g|_{g}^{2}= & \frac{(\mathcal{A}-A)^{2}}{A^{2}}+\frac{\left(\frac{q_{12}^{2}}{Q_{1}^{2}} \mathcal{A}+\mathcal{B}-B\right)^{2}}{B^{2}} \\
& +\frac{\left(\frac{q_{13}^{2}}{Q_{1}^{2}} \mathcal{A}+\frac{\left(q_{11} q_{23}-q_{12} q_{13}\right)^{2}}{Q_{2}^{2}} \mathcal{B}+\mathcal{C}-C\right)^{2}}{C^{2}}+2 \frac{\left(\frac{q_{12}}{Q_{1}} \mathcal{A}\right)^{2}}{A B} \\
& +2 \frac{\left(\frac{q_{13}}{Q_{1}} \mathcal{A}\right)^{2}}{A C}+2 \frac{\left(\frac{q_{12} q_{13}}{Q_{1}^{2}} \mathcal{A}+\frac{q_{11} q_{23}-q_{12} q_{13}}{Q_{2}} \mathcal{B}\right)^{2}}{B C} \\
& \doteqdot T_{1}+T_{2}+T_{3}+2 T_{4}+2 T_{5}+2 T_{6}
\end{aligned}
$$

converges to 0 as $t \rightarrow \infty$.

To see that $[g]$ is at least 1 -dimensional, choose any $\lambda>0$ and verify that $|h-g|_{g}^{2} \rightarrow 0$ when

$$
q_{\beta}=\left(\begin{array}{ccc}
\lambda A_{0} / C_{0} & & \\
& \lambda B_{0} / C_{0} & \\
& & \lambda
\end{array}\right) .
$$


To see that $[g]$ is at most 1-dimensional, suppose $|h-g|_{g}^{2} \rightarrow 0$. Since

$$
T_{4}=\frac{\left(q_{12} \frac{\mathcal{A}}{Q_{1}}\right)^{2}}{A B} \rightarrow q_{12}^{2}\left(\frac{C_{0}^{2} Q_{2}^{2}}{A_{0} B_{0} Q_{3}^{2}}\right)
$$

and

$$
T_{5}=\frac{\left(q_{13} \frac{\mathcal{A}}{Q_{1}}\right)^{2}}{A C} \rightarrow q_{13}^{2}\left(\frac{C_{0} Q_{2}^{2}}{A_{0} Q_{3}^{2}}\right),
$$

we have $q_{12}=q_{13}=0$. Then since

$$
T_{6}=\frac{\left(q_{23} \frac{Q_{1} \mathcal{B}}{Q_{2}}\right)^{2}}{B C} \rightarrow q_{23}^{2}\left(\frac{C_{0} Q_{2}^{2}}{B_{0} Q_{3}^{2}}\right),
$$

we have $q_{23}=0$ and can thus compute

$$
|h-g|_{g}^{2} \rightarrow\left(1-\frac{C_{0}}{A_{0}} \frac{q_{11}}{q_{33}}\right)^{2}+\left(1-\frac{C_{0}}{B_{0}} \frac{q_{22}}{q_{33}}\right)^{2} .
$$

So any choice of a single diagonal element $q_{i i}$ determines the remaining two.

\subsection{The geometry of $\mathbb{H}^{2} \times \mathbb{E}^{1}$.}

Let

$$
\mathcal{X}=\mathbb{R} \times \mathcal{H}^{2}=\left\{(w, x, y): w \in \mathbb{R},(x, y) \in \mathcal{H}^{2}\right\}
$$

and

$$
\mathcal{G}=\operatorname{Isom}(\mathbb{R}) \times \mathcal{I}^{2}=\left\{\left(\sigma_{s}, \tau_{u} \rho_{v}\right): s \in \mathbb{R},(u, v) \in \mathcal{H}^{2}\right\},
$$

where $\sigma_{s}$ is the translation $\sigma_{s}(w)=w+s$. Then $\mathcal{G}$ acts transitively on $\mathcal{X}$ by

$$
\left(\sigma_{s}, \tau_{u} \rho_{v}\right):(w, x, y) \mapsto\left(\sigma_{s}(w),\left(\tau_{u} \rho_{v}\right)(x, y)\right)=(w+s, v x+u, v y) .
$$

To define the 6-parameter family of homogeneous metrics having the geometry of $\mathbb{H}^{2} \times \mathbb{E}^{1}$, it is easiest to use local coordinates. Let $\beta=$ $\left(\partial / \partial z^{1}, \partial / \partial z^{2}, \partial / \partial z^{3}\right)$ be a basis for $T_{(w, x, y)} \mathcal{X}$ induced by coordinates 
$z^{1}, z^{2}, z^{3}$. Let $p$ be an arbitrary scalar product on $\mathbb{R}^{3}$, say $p_{\beta}=\left(p_{i j}\right), p_{\beta}^{-1}=$ $\left(p^{i j}\right)$, and define $g$ at the point $(w, x, y)$ by

$$
g=\left(\begin{array}{ccc}
p_{11} & p_{12} / y & p_{13} / y \\
p_{12} / y & p_{22} / y^{2} & p_{23} / y^{2} \\
p_{13} / y & p_{23} / y^{2} & p_{33} / y^{2}
\end{array}\right)
$$

Since

$$
D\left(\sigma_{s}, \tau_{u} \rho_{v}\right)=\left(\begin{array}{ccc}
1 & & \\
& v & \\
& & v
\end{array}\right)
$$

it is easily checked that each element of $\mathcal{G}$ is an isometry. Assuming as before that $\partial y / \partial z^{i}=\delta_{i 3}$, we find that the sectional curvatures of $g$ are

$$
\begin{aligned}
& K\left(\frac{\partial}{\partial z^{1}} \wedge \frac{\partial}{\partial z^{2}}\right)=\frac{p_{12}^{2}}{4} \cdot \frac{p^{33}}{p_{11} p_{22}-p_{12}^{2}} \\
& K\left(\frac{\partial}{\partial z^{1}} \wedge \frac{\partial}{\partial z^{3}}\right)=\frac{p_{12}^{2}}{4} \cdot \frac{p^{22}}{p_{11} p_{33}-p_{13}^{2}} \\
& K\left(\frac{\partial}{\partial z^{2}} \wedge \frac{\partial}{\partial z^{3}}\right)=\frac{p_{12}^{2}}{4} \cdot \frac{p^{11}}{p_{22} p_{33}-p_{23}^{2}}-\frac{p_{22}}{p_{22} p_{33}-p_{23}^{2}}
\end{aligned}
$$

and its Ricci tensor at the point $(w, x, y)$ is

$$
\operatorname{Rc}=\frac{p_{12}^{2}}{2}\left(\begin{array}{ccc}
p^{22} p^{33}-\left(p^{23}\right)^{2} & \left(p^{13} p^{23}-p^{12} p^{33}\right) / y & \left(p^{12} p^{23}-p^{13} p^{22}\right) / y \\
\left(p^{13} p^{23}-p^{12} p^{33}\right) / y & \left(p^{11} p^{33}-\left(p^{13}\right)^{2}\right) / y^{2} & \left(p^{12} p^{13}-p^{11} p^{23}\right) / y^{2} \\
\left(p^{12} p^{23}-p^{13} p^{22}\right) / y & \left(p^{12} p^{13}-p^{11} p^{23}\right) / y^{2} & \left(p^{11} p^{22}-\left(p^{12}\right)^{2}\right) / y^{2}
\end{array}\right)
$$

$$
+\frac{p_{22}}{y^{2}}\left(\begin{array}{ccc}
0 & & \\
& -p^{33} & p^{23} \\
& p^{23} & -p^{22}
\end{array}\right) .
$$

Notice that for this model, taking a minimal subgroup $\mathcal{G}<\operatorname{Isom}(\mathbb{R}) \times$ Conf $\left(\mathcal{H}^{2}\right)$ has allowed us to study more than just the usual product metrics. Now suppose $q$ is another scalar product, say $q_{\beta}=\left(q_{i j}\right), q_{\beta}^{-1}=\left(q^{i j}\right)$, 
and $h$ is the homogeneous metric defined at $(w, x, y)$ by

$$
h=\left(\begin{array}{ccc}
q_{11} & q_{12} / y & q_{13} / y \\
q_{12} / y & q_{22} / y^{2} & q_{23} / y^{2} \\
q_{13} / y & q_{23} / y^{2} & q_{33} / y^{2}
\end{array}\right) .
$$

As before, we shall study $[g]$ by letting $p$ and $q$ evolve by the Ricci flow; we may assume without loss of generality that $p_{\beta}$ is diagonal at $t=0$, say $p_{\beta}(0)=\left(\begin{array}{ccc}A_{0} & & \\ & B_{0} & \\ & & C_{0}\end{array}\right)$. Then $p_{\beta}$ remains diagonal and evolves by the system

$$
\frac{d}{d t} A=0, \quad \frac{d}{d t} B=2 \frac{B}{C}, \quad \frac{d}{d t} C=2
$$

with solution

$$
A=A_{0}, \quad B=B_{0}+2 \frac{B_{0}}{C_{0}} t, \quad C=C_{0}+2 t .
$$

Trivially, we have the conserved quantities

$$
A=A_{0} \quad \text { and } \quad \frac{B}{C}=\Phi \doteqdot \frac{B_{0}}{C_{0}} .
$$

Let $A$ be given by equation (64) and make a linear change of coordinates to induce the change of basis $\alpha=\beta A$ in $T_{(w, x, y)} \mathcal{X}$. Then $q_{\beta}$ becomes

$$
q_{\alpha}=\left(\begin{array}{ccc}
Q_{1} & & \\
& Q_{2} / Q_{1} & \\
& & Q_{3} / Q_{2}
\end{array}\right) \doteqdot\left(\begin{array}{ccc}
\mathcal{A}_{0} & & \\
& \mathcal{B}_{0} & \\
& & \mathcal{C}_{0}
\end{array}\right)
$$

where $Q_{n}>0$ is the determinant of the upper left $n \times n$ submatrix of $q_{\beta}=\left(q_{i j}\right)$. Hence the evolution of $h$ by the Ricci flow corresponds to

$$
\left(\begin{array}{ccc}
\mathcal{A} & & \\
& \mathcal{B} & \\
& & \mathcal{C}
\end{array}\right)=\left(\begin{array}{lll}
Q_{1} & & \\
& \frac{Q_{2}}{Q_{1}}+2 \frac{Q_{2}^{2}}{Q_{1} Q_{3}} t & \\
& & \frac{Q_{3}}{Q_{2}}+2 t
\end{array}\right) .
$$

Returning to the basis $\beta$, we calculate that $q_{\beta}=\left(A^{-1}\right)^{\operatorname{tr}} q_{\alpha} A^{-1}$ is the matrix given in (66). 
Theorem 3.2. The quasi-convergence class $[g]$ of an arbitrary metric $g$ having the geometry of $\mathbb{H}^{2} \times \mathbb{E}^{1}$ is exactly a 3-parameter family.

Proof. Note that $h \in[g]$ if and only iff every term in the sum

$$
|h-g|_{g}^{2}=T_{1}+T_{2}+T_{3}+2 T_{4}+2 T_{5}+2 T_{6}
$$

converges to 0 as $t \rightarrow \infty$, where $T_{1}, \ldots, T_{6}$ are defined in Theorem 3.1.

To see that $[g]$ is at most 3-dimensional, suppose $h \in[g]$ and note that $T_{3}, T_{4}$, and $T_{5}$ all converge to 0 for any choice of initial data. Since $T_{1} \rightarrow$ $\left(\frac{q_{11}}{A_{0}}-1\right)^{2}$, we must have

$$
q_{11}=A_{0} .
$$

Then since $T_{2} \rightarrow\left(\frac{C_{0} Q_{2}^{2}}{A_{0} B_{0} Q_{3}}-1\right)^{2}$, we get the relation

$$
Q_{3}=\frac{C_{0}}{A_{0} B_{0}} Q_{2}^{2} .
$$

And since $T_{6} \rightarrow\left(A_{0} q_{23}-q_{12} q_{13}\right)^{2} \frac{C_{0}}{A_{0} B_{0} Q_{3}}$, we have

$$
q_{23}=\frac{q_{12} q_{13}}{A_{0}} .
$$

Thus $q_{11}$ is determined by (a), $q_{23}$ is determined by (c) once $q_{12}$ and $q_{13}$ are known, and then $q_{33}$ is determined by (b) once $q_{22}$ is known.

To see that $[g]$ is at least 3-dimensional, let $\lambda>0$ and $\mu, \nu \in \mathbb{R}$ be given and take $\left(q_{i j}\right)$ to be

$$
\left(\begin{array}{ccc}
A_{0} & \mu & \nu \\
\mu & \frac{\lambda+\mu^{2}}{A_{0}} & \frac{\mu \nu}{A_{0}} \\
\nu & \frac{\mu \nu}{A_{0}} & \frac{\lambda C_{0}}{A_{0} B_{0}}+\frac{\nu^{2}}{A_{0}}
\end{array}\right) .
$$

Then $Q_{1}=A_{0}, Q_{2}=\lambda$, and $Q_{3}=\lambda^{2} A_{0}^{-1} B_{0}^{-1} C_{0}$, so $\left(q_{i j}\right)$ is positive definite. And the calculations above show clearly that $|h-g|_{g}^{2} \rightarrow 0$. 


\section{References.}

[1] R. Bryant, An introduction to Lie groups and symplectic geometry, in 'Geometry and Quantum Field Theory' (D. Freed and K. Uhlenbeck, eds.), IAS Park City mathematics series, 1, AMS, (1995).

[2] M. Eastham, The asymptotic solution of linear differential systems: Applications of the Levinson theorem, Clarendon Press, Oxford, (1989).

[3] R. Hamilton, Non-singular solutions of the Ricci flow on three-manifolds, Comm. Anal. Geom., to appear.

[4] R. Hamilton and J. Isenberg, Quasi-convergence of Ricci flow for a class of metrics, Comm. Anal. Geom., 4(1) (1993), 543-559.

[5] J. Isenberg and M. Jackson, Ricci flow of locally homogeneous geometries on closed manifolds, J. Differential Geom., 35 (1992), 723-741.

[6] D. Knopf, Quasi-convergence of the Ricci flow, Comm. Anal. Geom., to appear.

[7] J. Milnor, Curvatures of left invariant metrics on Lie groups, Adv. Math., 21 (1976), 293-329.

[8] P. Scott, The geometries of 3-manifolds, Bull. London Math. Soc., 15 (1983), 401-487.

[9] I.M. Singer, Infinitesimally homogeneous spaces, Comm. Pure Appl. Math., 13 (1960), 685-697.

[10] W. Thurston, Three dimensional manifolds, Kleinian groups, and hyperbolic geometry, Bull. Amer. Math. Soc., 6(3) (1982), 357-381.

E-mail address: knopf@math.wisc.edu

E-mail address: kevinm@uwm.edu

RECEIVED June 16, 1999. 